Discrete Comput Geom 31:83-124 (2004)

DOI: $10.1007 / \mathrm{s} 00454-003-2950-5$

\title{
Polyhedral Voronoi Diagrams of Polyhedra in Three Dimensions*
}

\author{
Vladlen Koltun ${ }^{1}$ and Micha Sharir ${ }^{1,2}$ \\ ${ }^{1}$ School of Computer Science, Tel Aviv University, \\ Tel Aviv 69978, Israel \\ \{vladlen,michas\}@tau.ac.il \\ ${ }^{2}$ Courant Institute of Mathematical Sciences, New York University, \\ New York, NY 10012, USA
}

\begin{abstract}
We show that the complexity of the Voronoi diagram of a collection of disjoint polyhedra in general position in 3-space that have $n$ vertices overall, under a convex distance function induced by a polyhedron with $O(1)$ facets, is $O\left(n^{2+\varepsilon}\right)$, for any $\varepsilon>0$. We also show that when the sites are $n$ segments in 3-space, this complexity is $O\left(n^{2} \alpha(n) \log n\right)$. This generalizes previous results by Chew et al. [10] and by Aronov and Sharir [4], and solves an open problem put forward by Agarwal and Sharir [2]. Specific distance functions for which our results hold are the $L_{1}$ and $L_{\infty}$ metrics. These results imply that we can preprocess a collection of polyhedra as above into a near-quadratic data structure that can answer $\delta$-approximate Euclidean nearest-neighbor queries amidst the polyhedra in time $O(\log (n / \delta))$, for an arbitrarily small $\delta>0$.
\end{abstract}

\section{Introduction}

The Voronoi diagram of a set $\Gamma$ of disjoint objects ("sites") in some space under some metric (or distance function) is a subdivision of the space into cells, one cell per site, such that the cell associated with a site $O \in \Gamma$ comprises the points in space for which $O$ is closer (under the given metric) than all other sites of $\Gamma$.

The study of Voronoi diagrams from the combinatorial and algorithmic points of view has a long and rich history in computational geometry, beginning with the very papers that launched this field in the 1970s [26] (see [25] for historical background). Following

* Work on this paper has been supported by a grant from the Israel Science Fund (for a Center of Excellence in Geometric Computing). Work by Micha Sharir was also supported by NSF Grants CCR-97-32101 and CCR00-98246, by a grant from the U.S.-Israel Binational Science Foundation and by the Hermann MinkowskiMINERVA Center for Geometry at Tel Aviv University. 
the intensive research conducted since, properties of Voronoi diagrams in the plane are very well understood, with respect to many different distance functions and types of sites (see, e.g., [6], [15], and [23] for recent comprehensive surveys of the subject).

In higher dimensions, however, some very basic problems concerning Voronoi diagrams are still wide open, and have withstood repeated attacks. One such problem is determining the combinatorial complexity of the Voronoi diagram of a set of $n$ "simplyshaped" sites in 3-space under a simple metric, where the prevailing conjecture is that this complexity is near-quadratic, as suggested by the best-known lower bound. However, the best upper bound derived so far is $O\left(n^{3+\varepsilon}\right)$, for any $\varepsilon>0$ [28], and even this bound was quite non-trivial to obtain; it is a corollary to the result that states that the lower envelope of an arrangement of semi-algebraic functions of constant description complexity in $\mathbb{R}^{d+1}$ has complexity $O\left(n^{d+\varepsilon}\right)$, for any $\varepsilon>0$ [27]. Since the Voronoi diagram of semi-algebraic sites under a semi-algebraic metric (all of constant description complexity) in $d$ dimensions can be represented as such an envelope, as observed by Edelsbrunner and Seidel [14], this result implies that the complexity of such Voronoi diagrams is $O\left(n^{d+\varepsilon}\right)$, for any $\varepsilon>0$, as well. ${ }^{1}$

A special case of this conjecture, which is still open, is the case of the Voronoi diagram of $n$ lines in 3-space under the Euclidean metric. Two results with quite involved proofs lend it credence.

The first result is due to Agarwal and Sharir [3], who have proved that a cross section (defined below) of a three-dimensional Voronoi diagram of pairwise-disjoint polyhedral sites with a total of $n$ vertices under the Euclidean metric has complexity $O\left(n^{2+\varepsilon}\right)$, for any $\varepsilon>0$. A cross section at height $\alpha$ of a Voronoi diagram is the locus of points in $\mathbb{R}^{3}$ that lie at distance exactly $\alpha$ from their closest site. It is equivalent to the boundary of the union of the Minkowski sums of the sites with a ball (of the given metric) of radius $\alpha$.

The second result has been very recently obtained by the authors [21], who have shown that the Voronoi diagram of lines in 3-space under the Euclidean metric has complexity $O\left(n^{2+\varepsilon}\right)$, for any $\varepsilon>0$, if the lines have a constant number of distinct orientations.

An exception to the unfortunate state of affairs regarding Euclidean Voronoi diagrams in higher dimensions is the case of point sites, for which a tight bound of $\Theta\left(n^{\lceil d / 2\rceil}\right)$ for the complexity of the Voronoi diagram under the Euclidean metric in $\mathbb{R}^{d}$ is well known (see, e.g., [14]).

A different research avenue is to consider Voronoi diagrams under a "polyhedral distance function" induced by a convex polytope with a constant number of facets (see Section 2.1 for details). It is this direction that the current paper takes, and we refer to such diagrams as polyhedral Voronoi diagrams. The polyhedral distance functions include the well-known $L_{1}$ and $L_{\infty}$ metrics, and are also interesting due to the fact that the Euclidean ball can be approximated arbitrarily well by a convex polytope. This implies that any Euclidean Voronoi diagram can be approximated with an arbitrarily high degree of accuracy by a polyhedral diagram. The results presented in this paper concerning polyhedral Voronoi diagrams, as well as some results that were known beforehand, are markedly better than the parallel known results for the Euclidean case.

\footnotetext{
${ }^{1}$ We note that constants of proportionality in bounds of the form $O\left(n^{q+\varepsilon}\right)$ depend on $\varepsilon$, and generally tend to infinity as $\varepsilon$ approaches zero.
} 
A tight bound of $\Theta\left(n^{2}\right)$ has recently been presented by Icking and Ma [18] for the complexity of a polyhedral Voronoi diagram of $n$ points in $\mathbb{R}^{3}$. This followed earlier works by Tagansky [29], who has derived a bound of $O\left(n^{2} \log n\right)$ for this complexity, and by Boissonnat et al. [7] who have showed the $\Theta\left(n^{2}\right)$ bound for some special cases (see also [29]), and have also given a tight bound of $\Theta\left(n^{\lceil d / 2\rceil}\right)$ for the case of points in $d$ dimensions under the $L_{\infty}$ metric or under a distance function induced by a simplex.

Perhaps more significantly, in light of the state of the art in the Euclidean case, an upper bound of $O\left(n^{2} \alpha(n) \log n\right)$ for the polyhedral Voronoi diagram of $n$ lines in $\mathbb{R}^{3}$ was proved by Chew et al. [10], together with a lower bound of $\Omega\left(n^{2} \alpha(n)\right)$. This followed an earlier work of Chew [9] who showed a bound of $O\left(n^{2} \alpha(n)\right)$ for the Voronoi diagram of lines in $\mathbb{R}^{3}$ under a "distance function" induced by a two-dimensional polygon (note that, strictly speaking, this is not a well-defined distance function).

In conclusion to their paper, Chew et al. [10] put forward the problem of obtaining a near-quadratic upper bound for the complexity of the polyhedral Voronoi diagram of line segments, and, more generally, of pairwise-disjoint polygons and polyhedra in three dimensions. It has since been restated by Agarwal and Sharir in their survey [2, Open Problem 6(ii)]..$^{2}$

In this paper we settle this problem by proving a bound of $O\left(n^{2} \alpha(n) \log n\right)$ for the polyhedral Voronoi diagram of $n$ line segments in 3-space, and a bound of $O\left(n^{2+\varepsilon}\right)$, for any $\varepsilon>0$, for the polyhedral Voronoi diagram of a collection of disjoint polyhedra in 3 -space with $n$ vertices altogether. (The constant of proportionality is quartic in the number of facets of the polytope that defines the distance function.) This also significantly generalizes the result of Aronov and Sharir [4] who used fairly complicated topological arguments to show a near-quadratic bound for the complexity of one cross section (defined above) of such a diagram (see also [5]).

Our results can be applied to show that a collection of disjoint polyhedra in 3-space with $n$ vertices altogether can be preprocessed into a data structure of size $O\left(n^{2+\varepsilon} / \delta^{4}\right)$, for any $\varepsilon>0$, such that this data structure can answer $\delta$-approximate Euclidean nearestneighbor queries amidst the polyhedra in time $O(\log (n / \delta))$, for an arbitrarily small $\delta>0$. (That is, the query returns a site whose distance to the query point is at most $1+\delta$ times the distance to the nearest site.) To our knowledge, no such data structure with comparable performance was available before. For the case of point sites, a number of near-linear approximate nearest-neighbor data structures have been presented, see [17] and the references within.

We remark that our results can also be applied to construct a piecewise-linear $\delta$ approximatation of near-quadratic complexity, of the medial axis of a three-dimensional polyhedron. Approximate construction of the medial axis has received considerable attention, see, e.g., [12] and the references within. We do not discuss this application in detail.

Some of the basic techniques we employ are inspired by ideas introduced by Chew et al. [10]. We extensively utilize the probabilistic analysis method developed by Tagansky [29], commonly known as the Tagansky technique. We also rely on the technique of

\footnotetext{
${ }^{2}$ We are aware of a subsequent study by Chew of the case of segment sites and a distance function induced by a tetrahedron; as far as we know, this work has not been published.
} 
counting schemes, originally introduced by Halperin and Sharir [16], [27], and refined in [1], [19], and [24] (see [20] and [28] for more details concerning this technique).

The rest of the paper is organized as follows. In the next section we describe the basic properties of the problem, and then present, in Section 3, the techniques utilized in our solution. The case of segment sites is discussed in Section 4, and polyhedral sites are then handled in Section 5. We describe the application to nearest-neighbor searching in Section 6.

\section{Preliminaries}

\subsection{Definitions and a Reduction to Triangles}

Let $\mathcal{P}$ be a convex polytope in $\mathbb{R}^{3}$ with a constant number of vertices, such that $\mathcal{P}$ contains the origin in its interior. We refer to the origin as the center of $\mathcal{P}$. The distance function induced by $\mathcal{P}$ is denoted by $d_{\mathcal{P}}$, and the distance of any point $v \in \mathbb{R}^{3}$ from a (possibly infinite) set of points $S \subset \mathbb{R}^{3}$ under $d_{\mathcal{P}}$ is

$$
d_{\mathcal{P}}(v, S)=\inf \{t \geq 0:(v+t \mathcal{P}) \cap S \neq \emptyset\} .
$$

The Voronoi diagram $\operatorname{Vor}_{\mathcal{P}}(\Gamma)$ of a set $\Gamma$ of $m$ pairwise disjoint sites in 3-space is the subdivision of $\mathbb{R}^{3}$ into $m$ cells, one cell for each site of $\Gamma$, such that the cell $V(\gamma)$, for some $\gamma \in \Gamma$, is

$$
V(\gamma)=\left\{v \in \mathbb{R}^{3}: d_{\mathcal{P}}(v, \gamma) \leq d_{\mathcal{P}}\left(v, \gamma^{\prime}\right), \forall \gamma^{\prime} \in \Gamma \backslash\{\gamma\}\right\} .
$$

If $\Gamma$ is a set of points, segments, or piecewise linear surfaces, then each $V(\gamma)$ is a (not necessarily convex) polyhedron. The vertices (resp. edges, faces) of all $V(\gamma)$, for $\gamma \in \Gamma$, are the vertices (resp., the edges, the faces) of $\operatorname{Vor}_{\mathcal{P}}(\Gamma)$. The combinatorial complexity of $\operatorname{Vor}_{\mathcal{P}}(\Gamma)$ is the number of faces of all dimensions of $\operatorname{Vor}_{\mathcal{P}}(\Gamma)$.

Let $\Gamma^{\prime}$ be a collection of pairwise disjoint closed polyhedra in 3-space with $n$ vertices altogether. Throughout this paper we assume that $\Gamma^{\prime}$ is in general position with respect to the polytope $\mathcal{P}$ that induces the distance function. That is, no two vertices in the scene lie on a line that is parallel to one of the faces of $\mathcal{P}$, no homothetic copy of $\mathcal{P}$ touches more than four vertices of $\Gamma^{\prime}$ with its boundary, no line parallel to an edge of $\mathcal{P}$ intersects more than two edges of $\Gamma^{\prime}$, etc. This assumption can be satisfied by an infinitesimal perturbation of the polyhedra of $\Gamma^{\prime}$. This assumption is essential, as the complexity of $\operatorname{Vor}_{\mathcal{P}}(\Gamma)$ can reach $\Omega\left(n^{3}\right)$ when the sites are in a degenerate configuration [7, Theorem 7.1].

We also assume that $\mathcal{P}$ is simplicial, i.e., all of its faces are triangles. If it is not, we infinitesimally perturb the vertices of $\mathcal{P}$ to general position, and take $\mathcal{P}$ to be the convex hull of the perturbed vertices. $\mathcal{P}$ is now simplicial, and it is easy to see that the complexity of $\operatorname{Vor}_{\mathcal{P}}(\Gamma)$ cannot decrease as a result of this perturbation, assuming $\Gamma^{\prime}$ is in general position as above.

Consider the collection of polygons that are faces of the polyhedra in $\Gamma^{\prime}$, and triangulate each of those polygons. Denote the resulting collection of triangles by $\Gamma$. By Euler's theorem, $|\Gamma|=O(n)$, and it is clear that $\operatorname{Vor}_{\mathcal{P}}(\Gamma)$ is a refinement of $\operatorname{Vor}_{\mathcal{P}}\left(\Gamma^{\prime}\right)$, and thus has larger complexity. Moreover, it is possible to perform an infinitesimal perturbation 
of the triangles of $\Gamma$ that will bring them into general position with respect to each other, i.e., they will become pairwise disjoint (including their boundaries), non-parallel, etc. It is easy to verify that such perturbation does not reduce the complexity of $\operatorname{Vor}_{\mathcal{P}}(\Gamma)$.

This discussion implies that an asymptotic bound on the complexity of the polyhedral Voronoi diagram of a scene that comprises pairwise disjoint triangles in general position in $\mathbb{R}^{3}$ would carry over to polyhedral Voronoi diagrams of collections of polyhedra such as $\Gamma^{\prime}$. We thus only discuss the former setting in our analysis.

It is easy to see that $\operatorname{Vor}_{\mathcal{P}}(\Gamma)$ does not contain edges and facets that are not adjacent to a vertex. The complexity of $\operatorname{Vor}_{\mathcal{P}}(\Gamma)$ is thus proportional to the number of its vertices, and it is therefore sufficient to provide a bound on this quantity.

\subsection{Vertices of the Diagram}

Some of the concepts introduced in the remainder of this section are illustrated in Fig. 1.

Placements. Let $\Gamma$ be a collection of triangles in general position in $\mathbb{R}^{3}$. Consider a homothetic copy of $\mathcal{P}$. It has four degrees of freedom, three for the location of its center in $\mathbb{R}^{3}$ and one for its scale. (That is, we represent a homothetic copy $\mathbf{x}+\lambda \mathcal{P}$ by the quadruple $(\mathbf{x}, \lambda)$.) Any set of four parameters that specifies location and scale as above is called a placement of $\mathcal{P}$. A placement is said to be free if the interior of the corresponding copy of $\mathcal{P}$ is disjoint from all the sites of $\Gamma$.

Uni-Contacts. A placement can be such that $\partial \mathcal{P}$ touches a triangle of $\Gamma$. This can happen in several ways. We say that a uni-contact (sometimes referred to as just a "contact") occurs if a vertex of $\mathcal{P}$ touches the interior of some triangle (a $V$-contact), or if the relative interior of an edge of $\mathcal{P}$ touches the relative interior of an edge of the triangle (an $E$-contact), or if the interior of a face of $\mathcal{P}$ touches a vertex of the triangle

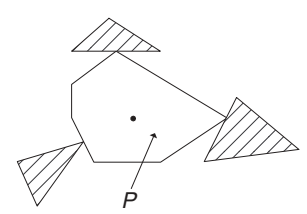

(a)

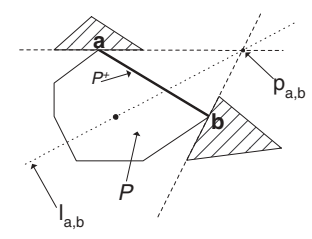

(d)

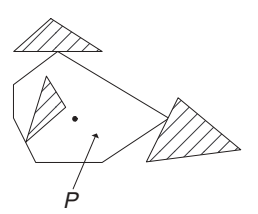

(b)

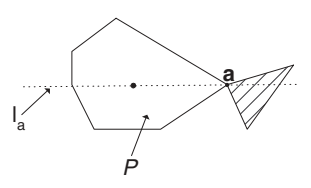

(e)

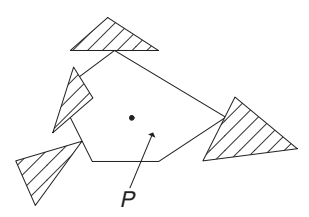

(c)

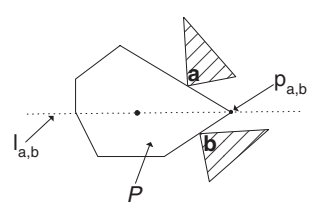

(f)

Fig. 1. An illustration of some of the definitions. Details are given in Section 2.5. 
(an $F$-contact). Instead of saying that a contact occurs, we sometimes say that $\mathcal{P}$ makes or maintains this contact. Notice that if we force $\mathcal{P}$ to maintain a certain (uni-)contact, it retains three degrees of freedom. For instance, fixing the location of the center of $\mathcal{P}$ and forcing $\mathcal{P}$ to make a certain contact uniquely determines the scale of $\mathcal{P}$.

Multi-Contacts. A bi-contact is said to occur if a vertex of $\mathcal{P}$ touches an edge of some triangle of $\Gamma$ (a $V^{2}$ bi-contact), or if an edge of $\mathcal{P}$ touches a vertex of the triangle (an $E^{2}$ bi-contact). Forcing $\mathcal{P}$ to maintain a bi-contact leaves it with only two degrees of freedom. A tri-contact is said to occur if a vertex of $\mathcal{P}$ touches a vertex of a triangle, and we denote this tri-contact by $V^{3}$. Maintaining such a tri-contact leaves $\mathcal{P}$ with only one degree of freedom - its center can only move along a line (more accurately, a half-line) in $\mathbb{R}^{3}$ that emanates from the vertex of $\Gamma$ involved in the contact.

Events. Certain placements of $\mathcal{P}$ can make more than one contact. An event is a placement of $\mathcal{P}$ in which it makes such contacts that together leave it with zero degrees of freedom. That is, any infinitesimal movement of $\mathcal{P}$ in any direction away from an event necessarily results in losing one of the contacts that are involved in the event. An event is said to be free if it is a free placement. An event is said to be a uni-contact event if $\mathcal{P}$ only makes uni-contacts in this placement, and it is said to be a multi-contact event otherwise.

Observation 1. There is a one-to-one correspondence between vertices of $\operatorname{Vor}_{\mathcal{P}}(\Gamma)$ and free events of $\mathcal{P}$ among $\Gamma$.

Freedom and Levels. A contact is said to be clean if the triangle of $\Gamma$ that is incident to $\partial \mathcal{P}$ in this contact is disjoint from the interior of $\mathcal{P}$. Otherwise, the contact is said to be dirty. An event is said to be pseudo-free if one or more of the contacts involved are dirty, but no triangle that is not involved in a contact intersects $\mathcal{P}$. A $k$-level free (resp., pseudo-free) event is an event that would be free (resp., pseudo-free) had exactly $k$ triangles been removed from $\Gamma$, but is not free if any set of triangles of size less than $k$ is removed, for some $k \geq 1$. An ( $\leq k)$-level event is an event whose level is at most $k$. An event is said to be a 0-level event if it is either free or pseudo-free. A property that will be highly useful in our analysis is that the number of 1-level events (and, in more generality, of all events whose level is bounded by a constant) of some specific combinatorial type (see below) is asymptotically bounded by the number of 0-level events of this type [11]. Details on types of events are given in Section 2.4 .

Degrees. Consider a placement of $\mathcal{P}$ that is an event. The degree of a vertex $v$ of $\mathcal{P}$ in this event is defined as the number of distinct contacts that involve either $v$ or an edge or a face of $\mathcal{P}$ that are incident to $v$. The degree of an edge $e$ of $\mathcal{P}$ is defined as the number of contacts that involve the interiors of $e$ or of the two faces of $\mathcal{P}$ that are incident to $e$. The degree of a face $f$ of $\mathcal{P}$ is defined as the number of contacts that involve the interior of $f . V-, V^{2}$, and $V^{3}$-contacts increase the degree of one vertex; $E$ - and $E^{2}$-contacts increase the degree of an edge and the two vertices adjacent to it; $F$-contacts increase the degree of a face, along with the degrees of the three edges and the three vertices 
adjacent to it. Notice that the general position assumption implies that the degree of a face is at most 1 . The degree of an edge or a vertex can reach 4 , which is the maximum possible degree, as is again implied by the general position assumption.

Incidence. Consider a placement of $\mathcal{P}$ that is an event. Two contacts $\alpha$ and $\beta$ are said to be incident in one vertex (resp., two vertices) in this event if the feature of $\mathcal{P}$ that is involved in $\alpha$ shares one vertex (resp., two vertices) with the feature of $\mathcal{P}$ that is involved in $\beta$.

Activeness. Let $i$ be the number of vertices of $\mathcal{P}$. We say that features of $\mathcal{P}$ that have strictly positive degree in some event are "active" in that event. The general position assumption implies that not more that four distinct contacts can participate in an event. Since each contact increases only the degrees of the vertices that are adjacent to the feature of $\mathcal{P}$ that is involved in this contact, and each feature (face, edge, or vertex) is adjacent to at most three vertices, at most 12 vertices can be active in an event. This implies that it suffices to consider polytopes $\mathcal{P}$ with 12 or fewer vertices. Indeed, if $\mathcal{P}$ has more than 12 vertices, then any event of $\mathcal{P}$ is also an event of a sub-polytope $\mathcal{Q}$ of $\mathcal{P}$ that has 12 or fewer vertices. ( $\mathcal{Q}$ is the convex hull of the active vertices; clearly, if a placement of $\mathcal{P}$ is free, then the corresponding placement of $\mathcal{Q}$ is also free.)

The maximal number of vertices of $\operatorname{Vor}_{\mathcal{P}}(\Gamma)$ is thus proportional to the maximal number of vertices of $\operatorname{Vor}_{\mathcal{Q}}(\Gamma)$, where $\mathcal{Q}$ has 12 vertices. Moreover, the dependence on $i$ in the constant of proportionality is quartic, since it suffices to consider all subpolytopes $\mathcal{Q}$ of $\mathcal{P}$ that are defined by four or fewer distinct features of $\mathcal{P}$. The number of sub-polytopes with at most 12 vertices that have to be considered is therefore at most $O\left(i^{4}\right)$. Hence, $N_{i}=O\left(i^{4} N_{12}\right)$, for all $i>12$, where $N_{i}=N_{i}(\Gamma)$ denotes the maximum number of vertices of $\operatorname{Vor}_{\mathcal{P}}(\Gamma)$, for a given $\Gamma$, when $\mathcal{P}$ has $i$ vertices.

Furthermore, in order to bound the maximal number of events of a certain combinatorial type, it suffices to consider only polytopes $\mathcal{P}$ that have a number of vertices that is equal to the maximum possible number of active vertices in events of this type. For example, it is enough to consider tetrahedral $\mathcal{P}$ (four vertices) to bound the maximal number of events that involve four $V$-contacts.

\subsection{Sliding Along Three Contacts}

A basic paradigm that proves very useful in our analysis is that of "sliding" along three contacts.

Consider an $E$-contact $\alpha$ that involves an edge $e$ of some triangle of $\Gamma$ and an edge $e^{\prime}$ of $\mathcal{P}$. Let $\Pi_{\alpha}$ be the plane that contains $e$ and is parallel to $e^{\prime}$. "Sliding" $\mathcal{P}$ along $\alpha$ means moving it in some direction, such that the contact $\alpha$ is maintained throughout the movement. It is obvious that during such sliding, the edge $e^{\prime}$ of $\mathcal{P}$ that is involved in $\alpha$ has to move inside the plane $\Pi_{\alpha}$.

If $\alpha$ is an $F$-contact, involving a vertex $v$ of some triangle of $\Gamma$ and a face $f$ of $\mathcal{P}$, sliding is defined in an analogous way, where the plane $\Pi_{\alpha}$ is the plane that contains $v$ and is parallel to $f$, and $f$ has to move inside $\Pi_{\alpha}$. The case of a $V$-contact is analogous, and the plane $\Pi_{\alpha}$ is the plane incident to the triangle of $\Gamma$ that is involved in the contact. 
When $\mathcal{P}$ is sliding along one contact $\alpha$, it has three remaining degrees of freedom, two for moving along the plane $\Pi_{\alpha}$, and one more for scaling. If we require $\mathcal{P}$ to maintain two contacts, $\alpha$ and $\beta$, we leave it with only two degrees of freedom. It is easy to see that the center of $\mathcal{P}$ is confined to move inside a specific plane in $\mathbb{R}^{3}$, and that this plane is incident to the line $\Pi_{\alpha} \cap \Pi_{\beta}$.

Sliding $\Pi$ along three uni-contacts, $\alpha, \beta$, and $\delta$, means moving it in such a way that it maintains all three contacts during the movement. This movement has only one degree of freedom, and the center of $\Pi$ is confined to move along a specific line $l_{\alpha, \beta, \delta}$ that is incident to the point $p_{\alpha, \beta, \delta}=\Pi_{\alpha} \cap \Pi_{\beta} \cap \Pi_{\delta}$ (in general position, this intersection is indeed a single point).

This means that if we wish to slide $\Pi$ along three contacts as above, starting from a specific placement, we can do so in only two specific directions along $l_{\alpha, \beta, \delta}$. One of these directions brings $\mathcal{P}$ towards $p_{\alpha, \beta, \delta}$. Since $\mathcal{P}$ has to continue touching the planes $\Pi_{\alpha}, \Pi_{\beta}$, and $\Pi_{\delta}$ during the movement, $\mathcal{P}$ shrinks to a point if the sliding continues until the center of $\mathcal{P}$ reaches $p_{\alpha, \beta, \delta}$. However, at least two of the contacts are necessarily lost prior to this point, since the triangles of $\Gamma$ that define $\alpha, \beta$, and $\delta$ are disjoint and only at most one of them can be incident to $p_{\alpha, \beta, \delta}$. This implies that at some point during the sliding towards $p_{\alpha, \beta, \delta}, \mathcal{P}$ will reach the boundary of one of the triangles it touches (at which moment a bi-contact event occurs), and will lose the contact defined by this triangle completely if it continues sliding.

Because of the linearity of trajectories, the volume of $\Pi$ increases in one of the directions of the sliding, and decreases in the other. The above discussion implies that the volume decreases (in other words, $\mathcal{P}$ "shrinks") when $\mathcal{P}$ slides towards $p_{\alpha, \beta, \delta}$. (In general, $\mathcal{P}$ does not shrink "into itself", but sweeps new portions of space while shrinking.)

Consider the situation where we slide $\mathcal{P}$ along three contacts starting from an event $X$ defined by four contacts. The general position assumption implies that the fourth contact made by $\mathcal{P}$ in the event will be lost immediately after the beginning of the sliding. Moreover, because of the linearity of trajectories, in one of the directions of the sliding this fourth contact will penetrate $\mathcal{P}$, while in the other direction, the placement of $\mathcal{P}$ will initially be free.

Suppose $\mathcal{P}$ makes the contacts $\alpha, \beta$, and $\delta$ at some placement. Consider a face $f$ of $\mathcal{P}$, such that one of the open half-spaces defined by the plane incident to $f$ (at this placement) contains $p_{\alpha, \beta, \delta}$, but does not contain any point of $\mathcal{P}$. Define $\mathcal{P}^{+} \subseteq \partial \mathcal{P}$ as the collection of such faces $f$, together with the edges and vertices of $\mathcal{P}$ incident to these faces. (These are the features of $\mathcal{P}$ that $p_{\alpha, \beta, \delta}$ "sees" at this placement of $\mathcal{P}$.) Consider now a face $g$ of $\mathcal{P}$, such that one of the open half-spaces defined by the plane incident to $f$ contains neither $p_{\alpha, \beta, \delta}$ nor any point of $\mathcal{P}$. Define $\mathcal{P}^{-} \subseteq \partial \mathcal{P}$ as the collection of such faces $g$, together with the edges and vertices of $\mathcal{P}$ incident to these faces. (These are the features "hidden" from $p_{\alpha, \beta, \delta}$.)

Observe that if $p_{\alpha, \beta, \delta}$ lies on a plane that is incident to a face $h$ of $\mathcal{P}, h$ belongs neither to $\mathcal{P}^{+}$nor to $\mathcal{P}^{-}$. If $p_{\alpha, \beta, \delta}$ lies on a line that is incident to an edge $e$ of $\mathcal{P}$, the same holds for $e$. If the point $p_{\alpha, \beta, \delta}$ is incident to a vertex $v$ of $\mathcal{P}$, this also holds for $v$. Moreover, if $p_{\alpha, \beta, \delta}$ is incident to a vertex $v$ of $\mathcal{P}$, then $\mathcal{P}^{+} \equiv \emptyset$.

Observation 2. (a) $\mathcal{P}^{+}$and $\mathcal{P}^{-}$are the same for any placement of $\mathcal{P}$ that makes the contacts $\alpha, \beta$, and $\delta$. (b) If a new clean uni-contact is made by $\mathcal{P}$ while $\mathcal{P}$ slides towards $p_{\alpha, \beta, \delta}$ 
as above, such that the new contact involves a feature $t$ of $\mathcal{P}$ and a triangle $\gamma$ of $\Gamma$ that was disjoint from $\mathcal{P}$ immediately before the contact was made, then $t$ necessarily belongs to $\mathcal{P}^{+}$. If such a situation occurs while $\mathcal{P}$ slides away from $p_{\alpha, \beta, \delta}$, then $t$ belongs to $\mathcal{P}^{-}$.

We say that each face of $\mathcal{P}$ that belongs to $\mathcal{P}^{+}$is a frontier face, and that $\mathcal{P}^{+}$as a whole is the frontier, when we slide towards $p_{\alpha, \beta, \delta}$ as above. Symmetrically, each face of $\mathcal{P}$ that belongs to $\mathcal{P}^{-}$is a frontier face and $\mathcal{P}^{-}$is the frontier when we slide along $\alpha$, $\beta$, and $\delta$ in the opposite direction.

Shrinking $\mathcal{P}$ Into Itself. Consider a free placement of $\mathcal{P}$ that makes three uni-contacts $\alpha, \beta$, and $\delta$, all incident to a vertex $v$ of $\mathcal{P}$. It is easy to see that in this case $p_{\alpha, \beta, \delta}=v$, and consequently $\mathcal{P}^{+} \equiv \emptyset$. (A two-dimensional equivalent of this situation is illustrated in Fig. 1(f).) This implies that if we slide $\mathcal{P}$ towards $p_{\alpha, \beta, \delta}$, along $\alpha, \beta$, and $\delta, \mathcal{P}$ will not encounter new uni-contacts and no uni-contact event will occur to $\mathcal{P}$. (Informally, $\mathcal{P}$ shrinks "into itself".) However, as observed above, $\mathcal{P}$ will at some point reach a bicontact event. This implies that we can uniquely charge a free uni-contact event that has a vertex of degree 3 to a free bi-contact event.

\subsection{Notation}

We distinguish between several types of events, based on the type of contacts that are involved in the event. Each type is denoted mnemonically by listing the types of the involved contacts. For instance, an $F F E V$ event is an event with two $F$-contacts, an

$E$-contact, and a $V$-contact. Note that it can equivalently be called an $E F V F$ event, say.

Such types of events can have many combinatorially different sub-types. For instance, one EEEE event may have four active vertices (the minimum possible number), while another may have eight (the maximum possible number). Such combinatorially different sub-types of events will often get different treatment in our analysis, and it is thus essential to be able to distinguish between them in the text. However, for the sake of clarity in the exposition, we do not introduce a text-based notation to distinguish between them, but instead provide a symbolic illustration of each sub-type whose analysis is non-trivial (see Fig. 2 for an example), and refer to the relevant illustration when discussing a certain sub-type.

Each combinatorial type of event is represented for the purpose of illustration as a multi-hypergraph, whose vertices correspond to the vertices of $\mathcal{P}$ that are active in the event. This hypergraph may have edges that connect one, two, or three vertices, and such edges correspond respectively to $V-, E$-, and $F$-contacts. The degree of a vertex in the hypergraph is the same as the degree of the corresponding vertex of $\mathcal{P}$ in an event of the illustrated combinatorial type. The illustrations depict planar realizations of the above multi-hypergraphs, showing edges of degree 3 ( $F$-contacts) as filled triangles, edges of degree 2 ( $E$-contacts) as straight or curved segments, and edges of degree 1 ( $V$ contacts) as small circles. For a concrete example, refer to Fig. 8(f) that shows one of the combinatorially distinct types of $F F E V$ events. In this type, the $V$-contact is "isolated", while the two $F$-contacts lie on faces of $\mathcal{P}$ that share a vertex, and both $F$-contacts similarly share a vertex with the $E$-contact. 


\subsection{Illustration}

Figure 1 provides an illustration in a two-dimensional setting of some of the concepts introduced above. In the figure the polygon $\mathcal{P}$ is shown with its center marked by a thick dot. Notice that in two dimensions a uni-contact event has only three contacts, and $\mathcal{P}$ has one degree of freedom if it has to maintain two uni-contacts or one bi-contact. A free uni-contact event is shown in (a), while pseudo-free and 1-level free events are shown in (b) and (c), respectively. Although $\mathcal{P}$ has six vertices, only four are active in these events. In (d), $\mathcal{P}$ makes the contacts $a$ and $b$, and can maintain these two contacts while sliding its center along the line $l_{a, b}$. The thickened edge is the only part of $\partial \mathcal{P}$ in $\mathcal{P}^{+}$, and if $\mathcal{P}$ slides towards the point $p_{a, b}$ (while maintaining the contacts $a$ and $b$ ) and encounters a uni-contact event, the new third contact can only involve this edge. In (e), a bi-contact $a$ is shown, together with the line $l_{a}$ that $\mathcal{P}$ can slide its center along while maintaining this contact. In (f), $\mathcal{P}$ makes two contacts $a$ and $b$ that are incident to a vertex that has degree 2. In this case, $\mathcal{P}^{+}$is empty, and $\mathcal{P}$ cannot encounter new uni-contact events when sliding towards $p_{a, b}$; the first event encountered during such sliding is necessarily a multi-contact event. (Informally, when sliding towards $p_{a, b}, \mathcal{P}$ shrinks "into itself".)

\section{Techniques}

In this section we outline the techniques and arguments we employ repeatedly throughout the analysis. Let $\Gamma$ be a set of $n$ pairwise disjoint triangles in $\mathbb{R}^{3}$, in general position.

\subsection{Multi-Contact Events}

The proofs of the following two lemmas are similar to parts of the analysis of Chew et al. [10].

Lemma 3.1. The number of free and pseudo-free events that involve a $V^{3}$-contact is $O(n)$.

Proof. Consider a free event $X$ that involves a $V^{3}$-contact $\alpha$ that is defined by a vertex $v$ of a triangle of $\Gamma$ and a vertex $w$ of $\mathcal{P}$. We charge $X$ to the pair $(v, w)$, and argue that the charging is unique. To see this, consider shrinking $\mathcal{P}$ starting from $X$, while maintaining the contact $\alpha$, until $\mathcal{P}$ shrinks to a point. During the shrinking, $\mathcal{P}$ remains free and no other event is encountered (because $\mathcal{P}$ shrinks into itself). Moreover, due to general position, the remaining contact involved in $X$ is lost immediately as the shrinking begins. Thus, we can reverse this shrinking process and expand $\mathcal{P}$ while maintaining $\alpha$, starting from $\mathcal{P}$ being infinitesimal, and the first event that is encountered is necessarily $X$. This proves the uniqueness of the above charging. The lemma now follows since the number of pairs $(v, w)$ as above is $O(n)$. Pseudo-free events are handled similarly.

Lemma 3.2. The number of free and pseudo-free events that involve $a V^{2}$ - or an $E^{2}$ contact is $O\left(n^{2} \alpha(n)\right)$. 
Proof. Consider a specific bi-contact $\varphi$. The number of distinct such contacts is $O(n)$. We prove below that the number of events that involve $\varphi$ is $O(n \alpha(n))$, which implies the lemma. Assume that $\varphi$ is a $V^{2}$-contact; $E^{2}$-contacts are handled analogously. Let $v$ be the vertex of $\mathcal{P}$ and let $e=\left(e_{1}, e_{2}\right)$ be the edge of a triangle in $\Gamma$ that are involved in $\varphi$ (where $e_{1}$ and $e_{2}$ denote the endpoints of $e$ ).

Consider the locus $W_{\varphi}$ of the centers of the placements of $\mathcal{P}$ that make the contact $\varphi$. It is a planar half-slab that is bounded by $e$, and by the lines $l_{1}$ and $l_{2}$ that are the analogously defined loci for the $V^{3}$-contacts between $v$ and $e_{1}$, and between $v$ and $e_{2}$, respectively. For any point $p$ on $e$, let $l_{p}$ be a ray parallel to $l_{1}$ (and to $l_{2}$ ) that emanates from $p$ inside $W_{\varphi}$. Parametrize $l_{p}$ such that the parameter of a point $q \in l_{p}$ is the distance $d_{\mathcal{P}}(q, p)$ between $q$ and $p$ (see Section 2.1). In this $\left(p^{\prime}, q^{\prime}\right)$-parametrization of $W_{\varphi}$ (where the parameter $p^{\prime}$ determines the point $p$ on $e$, and the parameter $q^{\prime}$ determines a point along $\left.l_{p}\right), q^{\prime}$ will be considered the "vertical" direction.

Let $\psi \neq \varphi$ be another contact, and let $s_{\varphi, \psi}$ be the locus of the centers of the placements of $\mathcal{P}$ that make both of the contacts $\varphi$ and $\psi . s_{\varphi, \psi}$ is a line segment that lies on $W_{\varphi}$. Consider the collection of $O(n)$ such segments that correspond to all contacts $\psi$ as above. This collection defines an arrangement of segments on $W_{\varphi}$. Each free event that involves $\varphi$ corresponds to a vertex in the lower envelope of this arrangement, appropriately defined with respect to the above parametrization. This was already observed in [10], and follows from the fact that if $\mathcal{P}$ slides on $l_{p}$ towards $p$, for some $p \in e$, while maintaining the contact $\varphi, \mathcal{P}$ shrinks into itself. The complexity of the above lower envelope is $O(n \alpha(n))$ [28], and this implies the lemma. Pseudo-free events are handled similarly.

In view of Lemmas 3.1 and 3.2, we only consider uni-contact events in the remainder of this section.

\subsection{Popular Vertices}

A vertex $v$ of $\mathcal{P}$ is said to be popular in a certain event of $\mathcal{P}$ if its degree in this event is at least 3. A two-dimensional equivalent of a popular vertex is shown in Fig. 1(f). The proof of the following lemma is based on ideas introduced by Chew et al. [10].

Lemma 3.3. The number of free and pseudo-free events in which one of the vertices of $\mathcal{P}$ is popular is $O\left(n^{2} \alpha(n)\right)$.

Proof. Consider a free event $X$, in which a vertex $v$ of $\mathcal{P}$ is popular. Shrink $\mathcal{P}$ into itself, and uniquely charge $X$ to a free bi-contact event $Y$, as described at the end of Section 2.3. The lemma follows from the fact that the number of such events $Y$ is $O\left(n^{2} \alpha(n)\right)$, as shown in Lemma 3.2. Pseudo-free events are handled similarly.

\subsection{Popular Faces}

A face $f$ of $\mathcal{P}$ is said to be popular if each edge adjacent to it is either involved in an $E$-contact, or is incident to a face other than $f$ that is involved in an $F$-contact. A two-dimensional equivalent of a popular face is shown in Fig. 1(d). 
Lemma 3.4. The number of free events in which one of the faces of $\mathcal{P}$ is popular is $O\left(n^{2} \alpha(n)\right)$.

Proof. Consider a free event $X$, in which a face $f$ of $\mathcal{P}$ is popular. If one of the vertices incident to $f$ is popular, then the lemma follows from Lemma 3.3. We thus assume below that the three vertices adjacent to $f$ all have degree at most 2 . The popularity of the face $f$ implies that their degrees must all be equal to 2 . This implies that exactly three contacts involve edges of $f$ and faces adjacent to these edges. Let $\alpha, \beta$, and $\delta$ be these three contacts. Consider sliding along $\alpha, \beta$, and $\delta$, as described in Section 2.3. It is easy to see that in one of the two possible directions of the sliding, $f$ is the sole frontier face. Slide in this direction and consider the first event $Y$ encountered during the sliding. We charge $X$ to $Y$; as in the proof of Lemma 3.3, the charging is unique. $Y$ is either a bi-contact event or it is an event that involves $\alpha, \beta, \delta$, and a fourth contact that involves $f$ or one of the edges or vertices of $f$. In the latter case, $Y$ necessarily has a popular vertex (which is a vertex of $f$ ). The lemma thus follows from the bounds stated in Lemmas 3.2 and 3.3.

\subsection{Reduction to Point Sites}

Lemma 3.5. The number of free and pseudo-free FFFF events of $\mathcal{P}$ is $O\left(n^{2}\right)$.

Proof. Consider the set of vertices of the triangles in $\Gamma$. It is a set of $O(n)$ points in 3 -space. It is easy to see that each free $F F F F$ event uniquely corresponds to a vertex in the Voronoi diagram of this set of points, under the distance function induced by $\mathcal{P}$. A pseudo-free $F F F F$ event similarly corresponds to a vertex in this diagram whose level is at most 8 (each of the at most four triangles with a vertex involved in the contact can have up to two other vertices lying inside $\mathcal{P}$ ). The complexity of this diagram is $O\left(n^{2}\right)$ [18], and this is also a bound for the number of free and pseudo-free $F F F F$ events.

\subsection{Induction}

Let $N_{i}=N_{i}(\Gamma)$ denote the maximum number of vertices of $\operatorname{Vor}_{\mathcal{P}}(\Gamma)$ when $\mathcal{P}$ has $i$ vertices. It was observed in Section 2.2 (paragraph "Activeness") that it suffices to consider $i \leq 12$. The discussion in that section also implies that the number of events in which only $j<i$ vertices of $\mathcal{P}$ are active is $O\left(N_{j}\right)$, even if $\mathcal{P}$ has $i$ vertices.

Our basic approach to deriving a bound on $N_{12}$ is to use induction. We first prove a near-quadratic bound on $N_{4}$, for which it suffices to consider tetrahedral $\mathcal{P}$ 's. We then bound $N_{i}$ in terms of $N_{i-1}$, for $5 \leq i \leq 12$. One of the ways to achieve this will be to charge events with $i$ active vertices to events with at most $i-1$ active vertices (see, e.g., Lemmas 3.6 and 3.8).

\subsection{The SFC Technique, or Sliding Away from a Face Contact}

Consider a free uni-contact event $X$ in which a face $f$ of $\mathcal{P}$ is involved in an $F$-contact. Let $\alpha, \beta$, and $\delta$ be the other three contacts involved in $X$. Consider sliding along $\alpha, \beta$, 
and $\delta$. By definition, only one of the parts $\mathcal{P}^{+}$and $\mathcal{P}^{-}$can include $f$. If this part is $\mathcal{P}^{+}$, we slide away from $p_{\alpha, \beta, \delta}$, and if this part is $\mathcal{P}^{-}$, we slide towards $p_{\alpha, \beta, \delta}$. It is easy to see that $\mathcal{P}$ is in a free placement immediately after the beginning of the sliding. We charge $X$ to the first event $Y$ encountered during the sliding. $Y$ is necessarily free. Observe that during this sliding, the face $f$ cannot become involved in new $F$-contacts, as observed in Section 2.3. Therefore, the fourth contact in $Y$ does not involve the face $f$.

The SFC technique is useful when we want to charge an event that involves a particular $F$-contact to an event that does not involve an $F$-contact with the same face. Consider, for example, the following situation.

We say that an $F$-contact is isolated if it is incident to no other contact. Suppose $X$ involves an isolated $F$-contact, and denote the face of $\mathcal{P}$ involved in this contact by $f$. Use the SFC technique to slide away from this contact and charge $X$ to an event $Y$. We claim that $Y$ has fewer active vertices than $X$. Indeed, any contact that does not involve the face $f$ cannot increase the degree of all three vertices of $f$. Since the degree of these three vertices was 1 in $X$, at least one of these vertices has degree 0 in $Y$. This implies the following lemma.

Lemma 3.6. The number of free events of $\mathcal{P}$ that have an isolated $F$-contact is at most $N_{i-1}$, where $i$ is the number of vertices in $\mathcal{P}$.

\subsection{The SEC Technique, or Sliding Away from an Edge Contact}

Consider a free event $X$ in which a face $f$ of $\mathcal{P}$ is involved in an $F$-contact $\alpha$, and an edge $e$ of $f$ is involved in an $E$-contact. The degree of $e$ is thus at least 2. Let $\beta$ and $\delta$ be the other two contacts involved in $X$. The point $p_{\alpha, \beta, \delta}$ lies on the plane $\Pi_{\alpha}$ that is incident to the face $f$ (at this placement). Thus, the edge $e$ that lies inside this plane can belong to only one of the parts $\mathcal{P}^{+}$and $\mathcal{P}^{-}$(this holds for the other two edges of $f$ as well). Sliding along $\alpha, \beta$, and $\delta$, as in the previous subsection, we can uniquely charge $X$ to a free event $Y$ that involves the contacts $\alpha, \beta, \delta$, and a fourth contact that is not an $E$-contact with the edge $e$. It is also easy to see that this fourth contact cannot be an $F$-contact with the second face incident to $e$. The ability to perform such charging proves useful in several steps of our analysis.

\subsection{Pseudo-Free Events}

Let $i$ be the number of vertices in $\mathcal{P}$. Let $M_{i}$ denote the maximum number of pseudo-free events of $\mathcal{P}$ among $\Gamma$, over all $\mathcal{P}$ with $i$ vertices. Roughly speaking, the next lemma states that the number of pseudo-free events is approximately proportional to the number of free events.

Lemma 3.7. $M_{i}=O\left(N_{i}+n^{2} \alpha(n)\right)$.

Proof. We distinguish between different pseudo-free events based on the number of dirty contacts involved. Let $M_{i}^{(a)}$ be the maximum number of pseudo-free events of $\mathcal{P}$ among $\Gamma$, that have $a$ dirty contacts and $4-a$ clean ones, for $1 \leq a \leq 4$. Consider a 
pseudo-free event with only one dirty contact. Slide $\mathcal{P}$ on the three clean contacts, away from this event, as explained in Section 2.3. When the sliding commences, the triangle $T$ of $\Gamma$ that was involved in the dirty contact is piercing $\mathcal{P}$. Consider the first event that occurs after the beginning of the sliding. This can either be (a) a bi-contact event, in which one of the the uni-contacts we have been sliding on degenerates into a bi-contact, or (b) a uni-contact event which involves some new triangle $T^{\prime} \in \Gamma$, and does not involve $T$, or (c) a free uni-contact event that involves $T$ (this happens at the moment $T$ stops piercing $\mathcal{P}$ ), or (d) another pseudo-free event that involves $T$, similar to the one we have started from.

In case (a), we charge this 1-level bi-contact event, and the number of such events is $O\left(n^{2} \alpha(n)\right)$ (Lemma 3.2). In case (b), we can similarly charge the 1-level free uni-contact event we have reached, and the number of such events is $O\left(N_{i}\right)$. (Recall from Section 2.2 that the number of 1-level events of a certain combinatorial type is asymptotically bounded by the number of 0 -level events of this type.) In case (c), we have reached a 0 -level free event, and charge it as above; the number of such events is at most $N_{i}$. In case (d), we continue sliding in the same fashion, disregarding the pseudo-free event we have encountered. Obviously, such pseudo-free events involving the same triangle $T$ can only happen $O(1)$ times during the sliding before some event of one of the other types happens. This implies that each pseudo-free event with one dirty contact can be charged as above, and, as is easily verified, the charging is unique up to $O(1)$ events that are possibly charged to the same event.

We have in effect showed that $M_{i}^{(1)}=O\left(N_{i}+n^{2} \alpha(n)\right)$. The same argument can be repeated virtually verbatim to show that $M_{i}^{(2)}=O\left(M_{i}^{(1)}+n^{2} \alpha(n)\right)$, that $M_{i}^{(3)}=$ $O\left(M_{i}^{(2)}+n^{2} \alpha(n)\right)$, and that $M_{i}^{(4)}=O\left(M_{i}^{(3)}+n^{2} \alpha(n)\right)$. This implies the lemma.

\subsection{The LEM Technique, or Lower Envelopes Merging}

Let $i$ be the number of vertices in $\mathcal{P}$. Let $X$ be a uni-contact event that involves four (not necessarily distinct) features (each being a vertex, an edge, or a two-dimensional face) of $\mathcal{P}$, participating in distinct contacts. Suppose that two of these features are incident, and the other two are each incident to a vertex of degree 1 (which means that no other feature among these four is incident to this vertex). In this case $X$ is said to be a LEM event. For instance, an event of the type shown in Fig. 2(f) is a LEM event, since it has two $E$ contacts that are incident (at two vertices), and two other $E$ contacts (that are incident at only one vertex) that are each incident to one vertex of degree 1 . Similarly, events of all the types shown in Figs. 2(d)-(i) are LEM events. The proof of the following lemma is based on the proof of Lemma 3.5 of [10].

Lemma 3.8. The number of free and pseudo-free LEM events of $\mathcal{P}$ is $O\left(N_{i-1}\right)$.

Proof. The proof treats only free LEM events. Lemma 3.7 easily implies that it extends to pseudo-free events.

The definition of LEM events implies that each such event has two contacts, denoted by $\alpha$ and $\beta$, that are incident in a common vertex denoted by $O$ (note that $O$ need not be 
unique), and two other contacts, denoted by $c$ and $d$, that are each incident to vertices of degree 1, denoted by $V$ and $U$, respectively. Obviously, $O \neq U \neq V$, since otherwise the degree of $U$ and/or $V$ would be higher than 1 .

Fix two specific contacts $\alpha$ and $\beta$ (with a common vertex $O$ ), and two vertices $U$ and $V$ of $\mathcal{P}$. Consider the locus $W_{\alpha, \beta}$ of the centers of the placements of $\mathcal{P}$ that make the contacts $\alpha$ and $\beta$. Similarly to the locus $W_{\alpha}$ in the proof of Lemma 3.2, $W_{\alpha, \beta}$ is a planar half-slab that is bounded by the line $l=\Pi_{\alpha} \cap \Pi_{\beta}$ that passes through the vertex $O$, and by two parallel lines orthogonal to $l[10]$. Parametrize $W_{\alpha, \beta}$ as in the proof of Lemma 3.2.

Consider a polytope $\mathcal{P}_{U}$ (resp., $\mathcal{P}_{V}$ ) that is defined to be the convex hull of all the vertices of $\mathcal{P}$ other than $U$ (resp., $V$ ). Let $s_{\alpha, \beta, \gamma}$ be the locus of the centers of the placements of $\mathcal{P}_{U}$ that make the contacts $\alpha, \beta$, and some other contact $\gamma$. If $s_{\alpha, \beta, \gamma}$ is not empty, it is a line segment that lies on $W_{\alpha, \beta}$. Consider the collection of the $O(n)$ such segments that correspond to all contacts $\gamma$ that can be made by $P_{U}$. This collection defines an arrangement $\mathcal{A}_{\alpha, \beta, U}$ of segments on $W_{\alpha, \beta}$. A similar collection of segments, and its arrangement $\mathcal{A}_{\alpha, \beta, V}$, can be defined with respect to $\mathcal{P}_{V}$.

As in the proof of Lemma 3.2, each free event that involves $\alpha, \beta, U$, and $V$, corresponds to a vertex in the lower envelope of the arrangements $\mathcal{A}_{\alpha, \beta, U}$ and $\mathcal{A}_{\alpha, \beta, V}$. This lower envelope is essentially the lower envelope of two lower envelopes: the lower envelope of $\mathcal{A}_{\alpha, \beta, U}$ and the lower envelope of $\mathcal{A}_{\alpha, \beta, V}$. The standard theory of Davenport-Schinzel sequences (see, e.g., [28]) implies that the complexity of a lower envelope of two univariate lower envelopes $L_{1}$ and $L_{2}$ of segments is asymptotically bounded by the sum of the complexities of $L_{1}$ and $L_{2}$.

Therefore, if we denote the complexity of the above lower envelopes by $L(\alpha, \beta, U)$ and $L(\alpha, \beta, V)$, respectively, and define $N(\alpha, \beta, U, V)$ to be the number of LEM events that involve $\alpha, \beta, U$, and $V$, as above, then it holds that $N(\alpha, \beta, U, V)=O(L(\alpha, \beta, U)+$ $L(\alpha, \beta, V))$. Notice now that vertices of the lower envelope of $\mathcal{A}_{\alpha, \beta, U}$ correspond to free events of $\mathcal{P}_{U}$. The number of such vertices, over all $\alpha, \beta$, and $U$, is thus $O\left(N_{i-1}\right)$, since $\mathcal{P}_{U}$ has $i-1$ vertices. Similarly, $L(\alpha, \beta, V)$ is also $O\left(N_{i-1}\right)$. If we denote by $|L E M|$ the number of free LEM events of $\mathcal{P}$, then the above discussion implies that

$$
\begin{aligned}
|L E M| & =\sum_{\alpha, \beta, U, V} N(\alpha, \beta, U, V)=O\left(\sum_{\alpha, \beta, U} L(\alpha, \beta, U)+\sum_{\alpha, \beta, V} L(\alpha, \beta, V)\right) \\
& =O\left(N_{i-1}\right),
\end{aligned}
$$

which is precisely the assertion we have set out to prove.

\subsection{The Tagansky Technique}

Let $\Omega$ be a collection of 0-level events of some specific combinatorial type, and let the collection of 1-level events of this type be denoted by $\Omega^{(1)}$. Let $\Phi$ be another collection of events, such that $|\Phi|=O\left(n^{2} \alpha(n) \log ^{c} n\right)$, for some non-negative integer constant $c$, or that $|\Phi|=O\left(n^{2+\varepsilon}\right)$, for any $\varepsilon>0$ (these will be the bounds that we will encounter in our analysis). 
Assume that, given an event of $\Omega$, we can either (i) charge it to $i_{1}>0$ events of $\Omega^{(1)}$, such that the number of times that each event of $\Omega^{(1)}$ is charged in this fashion is at most $j_{1}$, and also charge it to $i_{2} \geq 0$ other events of $\Omega^{(1)}$, such that the number of times that each event of $\Omega^{(1)}$ is charged in this fashion is at most $j_{2}$; or (ii) charge it to an event of $\Phi$, such that the number of times that each event of $\Phi$ is charged in this fashion is bounded by a constant. The following lemma is directly implied by the work of Tagansky [30], [29], and its proof can be easily worked out by slight modifications of Tagansky's analysis.

Lemma 3.9. Under the assumptions made above:

- If $i_{1} / j_{1}+i_{2} / j_{2}=2$, then $|\Omega|=O\left(n^{2} \alpha(n) \log ^{c+1} n\right)$ (respectively, $|\Omega|=O\left(n^{2+\varepsilon}\right)$, for any $\varepsilon>0)$.

- If $i_{1} / j_{1}+i_{2} / j_{2}>2$, then $|\Omega|=O\left(n^{2} \alpha(n) \log ^{c} n\right)$ (respectively, $|\Omega|=O\left(n^{2+\varepsilon}\right)$, for any $\varepsilon>0)$.

\subsection{Counting Schemes}

The Tagansky technique is a refined version of an older probabilistic analysis technique due to Halperin and Sharir [16], [27] (see [28] for a detailed exposition of the basic technique as well as its extensions, and [19] for a recent advanced application of it). This so-called "counting scheme" approach implies the following result, which is a special case of the more general treatment reviewed, e.g., in [28]:

Lemma 3.10. Assume that, for any $1 \ll k \ll n$, each 0 -level event of some specific combinatorial type can be either (a) charged to $\Omega\left(k^{2}\right)(\leq k)$-level events of this type, such that each $(\leq k)$-level event is charged in this fashion at most $O(1)$ times, or $(b)$ charged to a $(\leq k)$-level event of some other combinatorial type, such that the number of 0 -level events of this type is known to be $O\left(n^{2+\varepsilon}\right)$, for any $\varepsilon>0$, and each such event is charged in this manner $O(k)$ times. Under these assumptions, the number of events of the discussed combinatorial type is $O\left(n^{2+\varepsilon}\right)$, for any $\varepsilon>0$.

\section{Segment Sites}

Theorem 4.1. The complexity of the Voronoi diagram of a set $\Gamma$ of $n$ segments in 3 -space, under a convex distance function induced by a polytope $\mathcal{P}$ with $q$ facets, is $O\left(q^{4} n^{2} \alpha(n) \log n\right)$, provided that the segments are in general position with respect to $\mathcal{P}$, as defined in Section 2.1.

Proof. Let $i$ be the number of vertices of $\mathcal{P}$. As described in Section 3.5, the proof of Theorem 4.1 proceeds by induction on $i$, through a series of lemmas. Recall that it suffices to confine ourselves to $i \leq 12$. We begin by obtaining a bound for $N_{2}$ (Lemma 4.2), and then work our way up to $N_{12}$ (Lemma 4.9). Notice that the only uni-contacts that occur are $F$ - and $E$-contacts. Recall that it is sufficient to bound the number of free events. 
A free or pseudo-free event (or a 1-level free or pseudo-free event) is said to be good if it is a multi-contact event or an $F F F F$ event, or if it contains a popular vertex or an isolated $F$-contact. A free event (or a 1-level free event) that contains a popular face is also said to be good. Events with isolated $F$-contacts are easily handled using Lemma 3.6, and the various results shown in Section 3 imply that the number of free and pseudo-free good events without isolated $F$-contacts is $O\left(n^{2} \alpha(n)\right)$. We thus do not treat good events explicitly below.

Lemma 4.2. The complexity of the Voronoi diagram of a set of $n$ segments in 3-space is $O\left(n^{2}\right)$ under a distance function induced by a segment, and is $O\left(n^{2} \alpha(n)\right)$ under a distance function induced by a triangle. ${ }^{3}$ In other words, $N_{2}=O\left(n^{2}\right)$ and $N_{3}=$ $O\left(n^{2} \alpha(n)\right)$.

Proof. It is easy to see that if $\mathcal{P}$ is a triangle (three vertices), all the events of $\mathcal{P}$ among $\Gamma$ either have a popular vertex or a multi-contact. A bound of $O\left(n^{2} \alpha(n)\right)$ on the complexity of $\operatorname{Vor}_{\mathcal{P}}(\Gamma)$ in these cases is thus implied by Lemmas 3.1-3.3. If $\mathcal{P}$ is a segment (two vertices), it is easy to see that, assuming general position, each event can be charged to a pair of segments of $\Gamma$. This easily implies a bound of $O\left(n^{2}\right)$.

Lemma 4.3. The complexity of the Voronoi diagram of a set of $n$ segments in 3-space is $O\left(n^{2} \alpha(n) \log n\right)$ under a distance function induced by a tetrahedron. In other words, $N_{4}=O\left(n^{2} \alpha(n) \log n\right)$.

Proof. Let $\mathcal{P}$ be a tetrahedron. Notice that all events of $\mathcal{P}$ that have at least one $F$ contact have at least one popular vertex. Indeed, observe that an $E$-contact raises the degree of two vertices of $\mathcal{P}$ by 1 , while an $F$-contact similarly raises the degree of three vertices. Thus, the sum of the degrees of the four vertices of $\mathcal{P}$ in an event with one $F$-contact and three other contacts that are either $E$ or $F$ is at least $3+2+2+2=9$. However, this necessarily means that one of the four vertices of $\mathcal{P}$ has degree at least 3 , and is therefore popular. Thus, the $O\left(n^{2} \alpha(n)\right)$ bound on the number of events that have at least one $F$-contact follows from Lemma 3.3. This bound also applies to the number of $E E E E$ events that have a popular vertex.

We now bound the number of EEEE events that are not good, and therefore have no popular vertices or faces. (A similar analysis was carried out by Chew et al. [10], but only for the case of line sites.) Figure 2 illustrates all the combinatorially distinct types of such events when $\mathcal{P}$ is a polyhedron with an arbitrary number of vertices. Only two of them, the ones shown in Figs. 2(a) and 2(b), can occur when $\mathcal{P}$ is a tetrahedron, since all the other types have at least five active vertices. We treat these two types of events using the Tagansky technique (see Section 3.10). We outline only the treatment of the Fig. 2(b) type below; the Fig. 2(a) type is treated in complete analogy.

Consider a free event $X$ that belongs to the type illustrated in Fig. 2(b). Choose three of the contacts involved in $X$. Denote these contacts by $\alpha, \beta$, and $\gamma$, and refer to the

\footnotetext{
${ }^{3}$ Strictly speaking, these are not well-defined distance functions. What the lemma actually analyzes is the number of free events of the underlying segment or triangle, and it should be interpreted only in this context.
} 


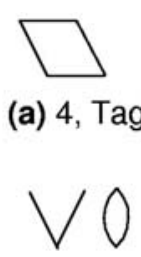

(f) 5 , LEM

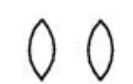

(b) 4 , Tag.

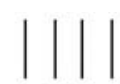

(c) 8 , Tag

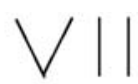

(d) 7, LEM

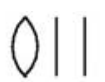

(e) 6, LEM

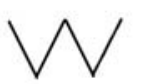

(g) 5, LEM

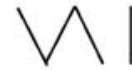

(h) 6, LEM

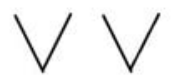

(i) 6, LEM

Fig. 2. Different combinatorial types of $E E E E$ events, not including good events. For each type, the number of active vertices and the name of the technique employed in the analysis are stated.

fourth contact as $\delta$. Slide on the contacts $\alpha, \beta$, and $\gamma$, such that the segment of $\Gamma$ that is involved in $\delta$ starts intersecting $\mathcal{P}$ immediately upon the beginning of the sliding. Charge $X$ to the first event that happens during this sliding, denoted by $Y$.

Denote the segments of $\Gamma$ involved in the contacts $\alpha, \beta, \gamma$, and $\delta$, by $A, B, C$, and $D$, respectively. Denote the vertices of $\mathcal{P}$ by $a, b, c$, and $d$, such that the edge of $\mathcal{P}$ involved in $\alpha$ and $\beta$ is $(a, b)$, and the edge involved in $\gamma$ and $\delta$ is $(c, d)$. All the vertices of $\mathcal{P}$ have degree 2 in the event $X$.

When the above sliding begins, the segment $D$ starts intersecting the interior of the faces $(a, c, d)$ and $(b, c, d)$, since they are the two faces adjacent to the edge $(c, d)$ that was in contact with $D$ in the event $X$. The first event that happens during the sliding may be a bi-contact event, in which case it is good. It may also be an event that involves the segment $D$, in one of the two fashions: either the segment $D$ reaches some edge of $\mathcal{P}$ or an endpoint of $D$ touches one of the faces $(a, c, d)$ and $(b, c, d)$. An event that involves an endpoint of $D$ in this way is a pseudo-free event with a popular vertex, since one of the vertices $a, b$ now has degree 3 . If the segment $D$ reaches an edge of $\mathcal{P}$, this edge must be an edge of one of the faces $(a, c, d),(b, c, d)$, and it cannot be the edge $(c, d)$, due to linearity of the trajectories. This edge thus has to be either $(c, a),(d, a),(c, b)$, or $(d, b)$. An event as above that involves a contact of $D$ with any of these edges is a pseudo-free event with a popular vertex, since in any of the listed cases, one of the vertices $a$ or $b$ becomes popular. Therefore, if the first event encountered during the sliding involves the segment $D$ or its endpoint, this event is a pseudo-free good event.

The event $Y$ may in addition be a uni-contact event that involves, aside from the contacts $\alpha, \beta$, and $\gamma$, an $F$-contact of some face of $\mathcal{P}$ with an endpoint of some segment $G \neq D$, or an $E$-contact of some edge of $\mathcal{P}$ that is not $(c, d)$ with some segment $G \neq D$. In all these cases it is easy to see that $Y$ is a 1-level event with a popular vertex.

The only case not considered above is that $Y$ involves an $E$-contact of some segment $G \neq D$ with the edge $(c, d)$. In this case, $Y$ is a 1 -level event of the same type as $X$. We have thus shown that either (i) $Y$ is a 1-level event of the same type as $X$, or (ii) $Y$ is a pseudo-free or a 1-level good event.

We repeat the described sliding and charging process for each of the four possible triples $(\alpha, \beta, \gamma)$. We show that each event $Y$ of type (i) is charged in this fashion at most twice.

Consider a 1-level event $Y$ of the type illustrated in Fig. 2(b), charged as above by some event $X$. Each event $X$ that could have charged $Y$ can be reached by sliding along 
some three contacts, away from $Y$, and considering the first encountered event. This event could have charged $Y$ if it is a 0-level free event of the type illustrated in Fig. 2(b), which implies that the segment $G$ that intersects the interior of $\mathcal{P}$ in the event $Y$ is involved in some contact in this event.

The segment $G$ intersects two faces of $\mathcal{P}$ in the event $Y$, which share an edge since $\mathcal{P}$ is a tetrahedron. It is clear that if this edge is not $(a, b)$ or $(c, d)$, then the event $Y$ cannot be charged by any event $X$. It is also clear that if this edge is $(a, b)$ or $(c, d)$, then the segment $G$ was involved in an $E$-contact with this edge in the event $X$. Assume that the edge is $(c, d)$, since the other possibility can be treated analogously.

In this case if we slide along the contacts $\alpha, \gamma$, and $\delta$ (denoted as above), an event that could have charged $Y$ as described above cannot be reached, and similarly if we slide along $\beta, \gamma$, and $\delta$. However, if we slide along $\alpha, \beta$, and $\gamma$, along the direction in which the segment involved in the contact $\delta$ does not penetrate $\mathcal{P}$, an event $X$ as described above can be reached. Analogously, we can reach such an event $X$ if we slide in a specific direction along $\alpha, \beta$, and $\delta$. Therefore, for any event $Y$, there are at most two potential events $X$ that can charge it. We have thus proved that each event $Y$ of type (i) is charged in the fashion described above at most twice. Also, it is easy to see that each event of type (ii) is charged at most a constant number of times.

Thus, we can either charge each event of the considered type to four 1-level events of this type, such that each event is charged at most twice, or to an event of a family whose size is $O\left(n^{2} \alpha(n)\right)$. Lemma 3.9 then implies that the number of events of the Fig. 2(b) type is $O\left(n^{2} \alpha(n) \log n\right)$. Since events of the Fig. 2(a) type can be treated analogously, this concludes the proof of the lemma.

Lemma 4.4. $\quad N_{5}=O\left(n^{2} \alpha(n) \log n\right)$.

Proof. Let $\mathcal{P}$ be a convex polyhedron with five vertices. Events of $\mathcal{P}$ with five active vertices are either good or belong to the types illustrated in Figs. 2(f), 2(g), 3(m), 3(n), 3(o), 4(y), and 4(z). Events of the types shown in Figs. 2(f) and 2(g) are LEM events, and their number is thus $O\left(N_{4}\right)=O\left(n^{2} \alpha(n) \log n\right)$ (Lemmas 3.8 and 4.3).

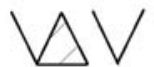

(a) 7, LEM

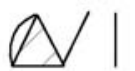

(f) 6 , LEM

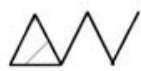

(k) 6, LEM

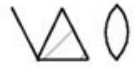

(b) 6, LEM

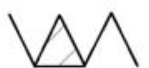

(g) 6, LEM

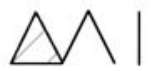

(I) 7 , LEM

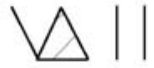

(c) 8 , LEM

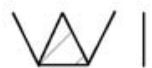

(h) 7, LEM

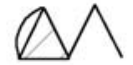

(m) 5, SEC

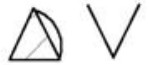

(d) 6, LEM

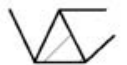

(i) 6 , LEM

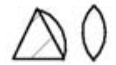

(n) 5 , SFC

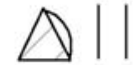

(e) 7, LEM

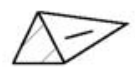

(j) 6 , LEM

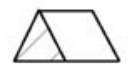

(o) 5 , SFC

Fig. 3. Different combinatorial types of $F E E E$ events, not including good events. For each type, the number of active vertices and the name of the technique employed in the analysis are stated. 


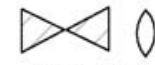

(a) 7, LEM

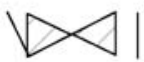

(f) 8, LEM

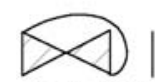

(k) 7, LEM

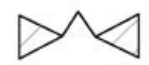

(p) 7, LEM

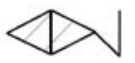

(u) 6, LEM

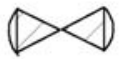

(z) 5 , SFC

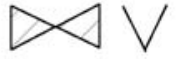

(b) 8, LEM

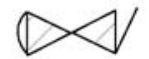

(g) 6, LEM

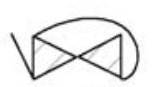

(I) 6, LEM

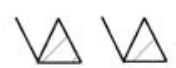

(q) 8 , LEM

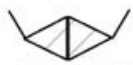

(v) 6, LEM

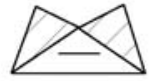

(c) 7, LEM

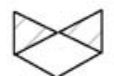

(h) 6, LEM

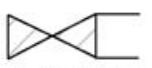

(m) 7, LEM

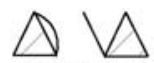

(r) 7, LEM

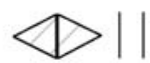

(w) 8 , LEM

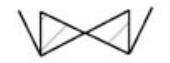

(d) 7, LEM

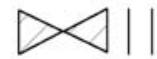

(i) 9 , LEM

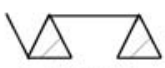

(n) 7, LEM

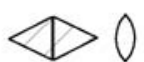

(s) 6, LEM

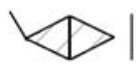

(x) 7, LEM

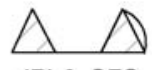

(B) 6 , SFC

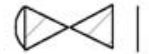

(e) 7, LEM

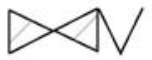

(j) 7, LEM

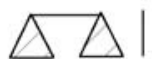

(o) 8, LEM

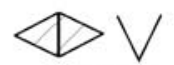

(t) 7, LEM

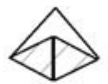

(y) 5 , SFC

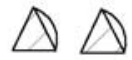

(A) 6, SFC

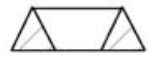

(C) 6 , SFC

Fig. 4. FFEE events. (Strictly speaking, events shown in (c) and (k) are combinatorially equivalent. The geometric realizations corresponding to these two illustrations are however quite different.)

Let $X$ be a free event of a type illustrated in Fig. 3(n) or 3(o). Let $f$ be the face of $\mathcal{P}$ that is involved in the $F$-contact in $X$. Use the SFC technique (Section 3.6) to slide away from this $F$-contact. Charge $X$ to the first event $Y$ encountered during the sliding. $Y$ is a free event, since the placement of $\mathcal{P}$ immediately upon the beginning of the sliding is free. The SFC technique implies that either $Y$ is a multi-contact event or it has the same triple of $E$-contacts as $X$, together with a fourth contact that is not an $F$-contact with $f$. It is easy to see that either (i) $Y$ has only four active vertices, or (ii) $Y$ is a good event, or (iii) $Y$ is a LEM event. Since the number of such events is $O\left(n^{2} \alpha(n) \log n\right)$, and any event $Y$ can be charged as above only a constant number of times, this implies that the number of events of the types illustrated in Figs. 3(n) and 3(o) is $O\left(n^{2} \alpha(n) \log n\right)$.

Let $X$ be a free event of the type illustrated in Fig. 3(m). Let $a$ denote the edge of $\mathcal{P}$ that is involved in the $E$-contact that is incident with two vertices to the $F$-contact. Use the SEC technique (Section 3.7) to slide away from this $E$-contact and charge $X$ to a free event $Y$, as above. The SEC technique implies that $Y$ cannot have an $E$-contact that involves $a$, or a second $F$-contact that involves a face incident to $a$. This implies that either (i) $Y$ is a good event, or (ii) $Y$ is an event of the type shown in Fig. 3(o). The number of events of the Fig. 3(m) type is therefore $O\left(n^{2} \alpha(n) \log n\right)$, as above.

Let $X$ be a free event of one of the types illustrated in Figs. 4(y) and 4(z). Use the SFC technique (Section 3.6) to slide away from one of the two $F$-contacts in $X$. Charge $X$ to the first event $Y$ encountered during the sliding. As above, $Y$ is a free event, and does not involve an $F$-contact with the same face. Thus, either (i) $Y$ is a good event, or (ii) $Y$ is an event of one of the types shown in Figs. 3(m), 3(n), and 3(o). The number of events of the types illustrated in Figs. 4(y) and 4(z) is thus $O\left(n^{2} \alpha(n) \log n\right)$. 


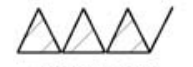

(a) 8 , LEM

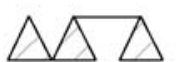

(e) 8 , LEM

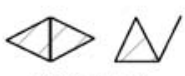

(i) 8 , LEM

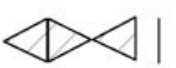

(m) 8 , LEM

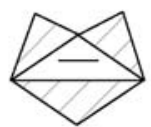

(p) 8 , LEM

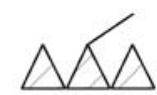

(b) 8, LEM

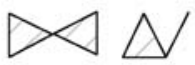

(f) 9, LEM
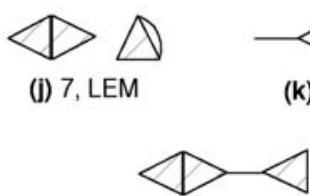

(n) 7, LEM

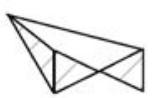

(q) $6, \mathrm{SFC}$

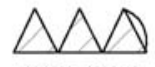

(c) 7, LEM

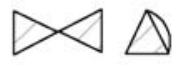

(g) 8, LEM

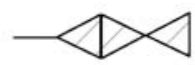

(k) 7, LEM

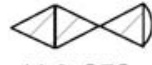

(r) 6 , SFC

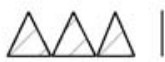

(d) 9, LEM

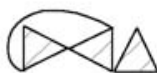

(h) 7, LEM

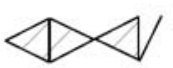

(I) 7, LEM

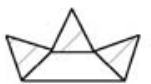

(o) 7, LEM

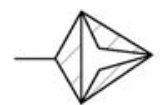

(s) 7, LEM

Fig. 5. FFFE events.

Lemma 4.5. $N_{6}=O\left(n^{2} \alpha(n) \log n\right)$.

Proof. Let $\mathcal{P}$ be a convex polyhedron with six vertices. Events of $\mathcal{P}$ with six active vertices are either good or belong to the types illustrated in Figs. 2(e), 2(h), 2(i), 3(b), 3(d), 3(f), 3(g), 3(i), 3(j), 3(k), 4(g), 4(h), 4(l), 4(s), 4(u), 4(v), 4(A), 4(B), 4(C), 5(q), and $5(\mathrm{r})$.

Free events of the types shown in Figs. 2(e), 2(h), 2(i), 3(b), 3(d), 3(f), 3(g), 3(i), $3(\mathrm{j}), 3(\mathrm{k}), 4(\mathrm{~g}), 4(\mathrm{~h}), 4(\mathrm{l}), 4(\mathrm{~s}), 4(\mathrm{u})$, and $4(\mathrm{v})$ are LEM events, and their number is thus $O\left(N_{5}\right)=O\left(n^{2} \alpha(n) \log n\right)$ (Lemmas 3.8 and 4.4).

Let $X$ be a free event of a type illustrated in Fig. 4(A), 4(B), or 4(C). Use the SFC technique to slide away from any one of the two $F$-contacts of $X$ and charge $X$ to a free event $Y$, as in the proof of Lemma 4.4. Either (i) $Y$ is good, or (ii) $Y$ is a LEM event, or (iii) $Y$ has at most five active vertices. The number of events of the types illustrated in Figs. 4(A), 4(B), and 4(C) is therefore $O\left(n^{2} \alpha(n) \log n\right)$.

Let $X$ be a free event of a type illustrated in Fig. 5(q) or 5(r). Use the SFC technique to slide away from the (unique) $F$-contact that involves a face that is incident to a vertex of degree 1. Charge $X$ to the first event $Y$ encountered during the sliding. In complete analogy to the above, the number of these events is $O\left(n^{2} \alpha(n) \log n\right)$. This completes the proof of the lemma.

Lemma 4.6. $\quad N_{7}=O\left(n^{2} \alpha(n) \log n\right)$.

Proof. Let $\mathcal{P}$ be a convex polyhedron with seven vertices. Events of $\mathcal{P}$ with seven active vertices are either good or belong to the types illustrated in Figs. 2(d), 3(a), 3(e), 
3(h), 3(l), 4(a), 4(c), 4(d), 4(e), 4(j), 4(k), 4(m), 4(n), 4(p), 4(r), 4(t), 4(x), 5(c), 5(h), 5(j), $5(\mathrm{k}), 5(\mathrm{l}), 5(\mathrm{n}), 5(\mathrm{o})$, and 5(s). All of these events are LEM events, and the number of free such events is thus $O\left(N_{6}\right)=O\left(n^{2} \alpha(n) \log n\right)$ (Lemmas 3.8 and 4.5).

Lemma 4.7. $N_{8}=O\left(n^{2} \alpha(n) \log n\right)$.

Proof. Let $\mathcal{P}$ be a convex polyhedron with eight vertices. Events of $\mathcal{P}$ with eight active vertices are either good or belong to the types illustrated in Figs. 2(c), 3(c), 4(b), 4(f), 4(o), 4(q), 4(w), 5(a), 5(b), 5(e), 5(g), 5(i), 5(m), and 5(p). All of these events except the Fig. 2(c) type are LEM events, and the number of free such events is $O\left(N_{7}\right)=O\left(n^{2} \alpha(n) \log n\right)$ (Lemmas 3.8 and 4.6).

We treat events of the type illustrated in Fig. 2(c) using the Tagansky technique (see Section 3.10). Consider a free event $X$ that belongs to this type. Choose three of the contacts involved in $X$. Denote these contacts by $\alpha, \beta$, and $\gamma$, and refer to the fourth contact as $\delta$. Slide on the contacts $\alpha, \beta$, and $\gamma$, such that the segment of $\Gamma$ that is involved in $\delta$ starts intersecting $\mathcal{P}$ immediately upon the beginning of the sliding. Charge $X$ to the first event that happens during this sliding, denoted by $Y$. We repeat the sliding and charging for each of the four possible triples $(\alpha, \beta, \gamma)$.

In complete analogy to the analysis in the proof of Lemma 4.3, we can argue that either (i) $Y$ is a 1-level event of the same type as $X$, or (ii) $Y$ is a pseudo-free or a 1-level LEM event, or (iii) $Y$ is a 1-level good event, or (iv) $Y$ is a pseudo-free or 1-level event with only 7 active vertices. Observe that the number of events $Y$ in cases (ii) and (iv) is $O\left(N_{7}\right)=O\left(n^{2} \alpha(n) \log n\right)$, and the number of events in case (iii) is $O\left(n^{2} \alpha(n)\right)$.

Again in analogy to the arguments in the proof of Lemma 4.3, we can show that each event $Y$ of type (i) is charged in the fashion described above at most once, and that the events of the other types are charged at most a constant number of times each. Lemma 3.9 thus implies that the number of events of the Fig. 2(c) type is $O\left(n^{2} \alpha(n) \log n\right)$.

Lemma 4.8. $\quad N_{9}=O\left(n^{2} \alpha(n) \log n\right)$.

Proof. Let $\mathcal{P}$ be a convex polyhedron with nine vertices. Events of $\mathcal{P}$ with nine active vertices are either good or belong to the types illustrated in Figs. 4(i), 5(d), and 5(f). All of these events are LEM events, and the number of free such events is $O\left(N_{8}\right)=$ $O\left(n^{2} \alpha(n) \log n\right)$ (Lemmas 3.8 and 4.5).

Lemma 4.9. $N_{d}=O\left(n^{2} \alpha(n) \log n\right)$, for $10 \leq d \leq 12$.

Proof. A free event of $\mathcal{P}$ with ten or more active vertices is either good (e.g., an $F F F F$ event) or has at least one isolated $F$-contact. The number of events of the latter kind is easily bounded by induction, using Lemma 3.6. Since $N_{9}=O\left(n^{2} \alpha(n) \log n\right)$ (Lemma 4.8), this implies the lemma.

This completes the proof of Theorem 4.1. 


\section{Polyhedral Sites}

Theorem 5.1. The complexity of the Voronoi diagram of a collection of pairwise disjoint polyhedral sites in 3-space that have $n$ vertices overall, under a convex distance function induced by a polytope $\mathcal{P}$ with $q$ facets, is $O\left(q^{4} n^{2+\varepsilon}\right)$, for any $\varepsilon>0$, provided that the sites are in general position with respect to $\mathcal{P}$, as defined in Section 2.1.

Proof. Recall from Section 2.1 that it is sufficient to analyze the complexity of $\operatorname{Vor}_{\mathcal{P}}(\Gamma)$ when $\Gamma$ is a set of $n$ pairwise disjoint triangles in $\mathbb{R}^{3}$, in general position. As in the preceding section, it is enough to obtain a bound on the number of free events. Events that were defined to be good in the last section will not be treated explicitly here as well, as their number is known to be $O\left(n^{2} \alpha(n)\right)$. Moreover, free $E E E E, E E E F, E E F F, E F F F$, and $F F F F$ events uniquely correspond to vertices in the Voronoi diagram of the set of the $3 n$ segments that are the edges of the triangles of $\Gamma$. The complexity of this diagram is $O\left(n^{2} \alpha(n) \log n\right)$ (Theorem 4.1), which bounds the number of free events of these types. We refer to these events as segment events. We only analyze non-segment events that are not good; in particular, such events involve at least one $V$-contact.

The Tagansky technique is used over and over in the course of the proof, and each time it is employed, it requires showing that the number $i$ of times that each 1-level event is charged is related in a certain way to the number $j$ of times each 0-level event charges (e.g., arguing that any 0-level event dispenses four "units of charge", but each 1-level event is charged at most twice), and also showing that the types of the events that are charged satisfy the requirements of Lemma 3.9. Since the arguments for showing this always follow the lines of the ones used for Lemmas 4.3 and 4.7, and since giving them in full would inflate the size of the paper exceedingly, we omit them and report the relevant types of events and the quantities $i$ and $j$ in each case without explicit proof.

As in the previous section, our analysis proceeds by induction, which is developed through a series of lemmas.

Lemma 5.2. The complexity of the Voronoi diagram of a set of $n$ triangles in 3-space is $O\left(n^{2}\right)$ under a distance function induced by a segment. In other words, $N_{2}=O\left(n^{2}\right)$.

Proof. Similarly to the proof of Lemma 4.2, it is easy to charge each free event to a pair of triangles of $\Gamma$, such that each pair is charged $O(1)$ times. This yields the desired bound.

Lemma 5.3. $\quad N_{3}=O\left(n^{2} \alpha(n) \log n\right)$.

Proof. Let $\mathcal{P}$ be a triangle. The events of $\mathcal{P}$ that we analyze belong to the types illustrated in Figs. 6(c), 6(o), and 7(f).

We treat events of the type illustrated in Fig. 6(c) using the Tagansky technique (see Section 3.10). Consider a free event $X$ that belongs to this type. Let $d$ be one of the two $V$-contacts involved in $X$. Denote the other three contacts by $a, b$, and $c$. Slide on the contacts $a, b$, and $c$, such that the triangle $\delta$ of $\Gamma$ that is involved in $d$ starts intersecting $\mathcal{P}$ immediately upon the beginning of the sliding. Charge $X$ to the first event that happens 


\section{$\bigvee_{0}^{\circ}$}

(a) 5, LEM

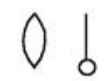

(f) 4, Tag.

$0_{\circ}^{\circ}$

(k) 4, LEM

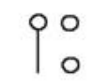

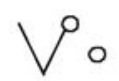

(b) 4, LEM<smiles>C1=CO1</smiles>

(g) 6, LEM

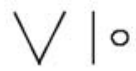

(I) 6, LEM

1000

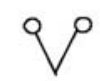

(c) 3, Tag.<smiles>C1=C2C=C1O2</smiles>

(h) 4, Tag.

010

(m) 5, LEM

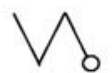

91

(d) 4, Tag.

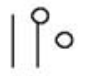

(i) 5, LEM<smiles>C=CC(=O)C=C</smiles>

(n) 7, Tag.

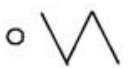

(s) 5, LEM

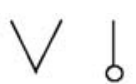

(e) 5, LEM

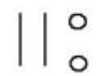

(j) 6, Tag .

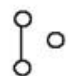

(o) 3, Tag.

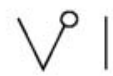

(t) 5 , LEM

Fig. 6. Different combinatorial types of EEEV, EEVV, and $E V V V$ events, not including good events.

during this sliding, denoted by $Y$. It can be shown that either (i) $Y$ is a 1-level event of the same type as $X$, or (ii) $Y$ is a 1-level or a pseudo-free good event. Repeat this sliding and charging process for each of the two possible choices of $d$. Note that the number of events $Y$ in case (ii) is $O\left(n^{2} \alpha(n)\right)$ (recall from Section 2.2 that the number of 1-level events of a specific type is asymptotically bounded by the number of 0-level events of this type).

Each event $Y$ of type (i) is charged in the fashion described above at most once, because the pair of edges of $\mathcal{P}$ that $\delta$ crosses at $Y$ determines $d$ uniquely, and each event of the other types is charged at most a constant number of times. Lemma 3.9 thus implies that the number of events of the Fig. 6(c) type is $O\left(n^{2} \alpha(n) \log n\right)$. Note that the above analysis also applies to pseudo-free events of the Fig. 6(c) type in which one or both of the $E$-contacts are dirty, but the $V$-contacts are clean.

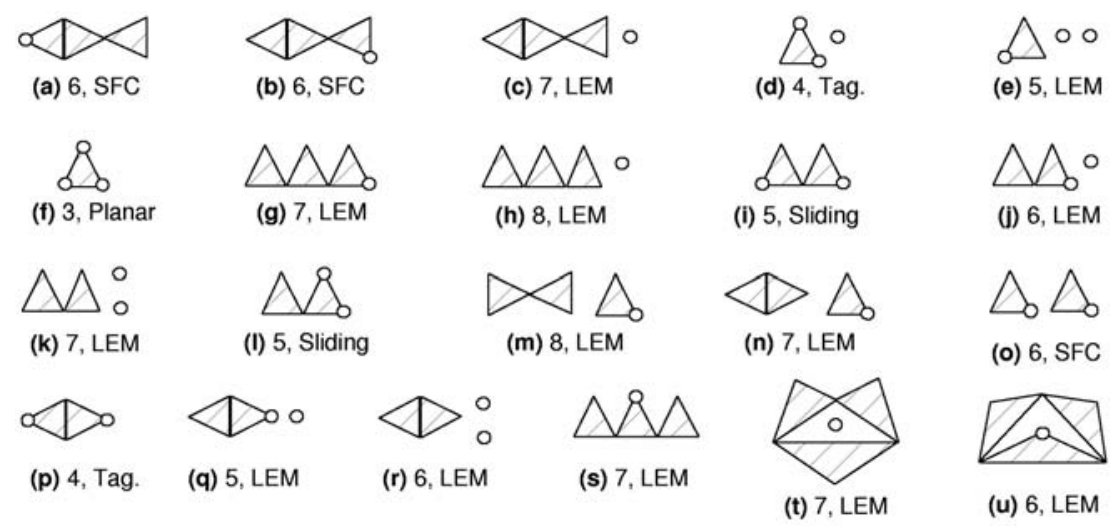

Fig. 7. Different combinatorial types of $F F F V, F F V V$, and $F V V V$ events, not including good events. 
We treat events of the type illustrated in Fig. 6(o) in a similar fashion. Consider a free event $X$ that belongs to this type. Let $d$ be one of the three $V$-contacts involved in $X$. Denote the other three contacts by $a, b$, and $c$. Slide on the contacts $a, b$, and $c$, such that the triangle of $\Gamma$ that is involved in $d$ starts intersecting $\mathcal{P}$ immediately upon the beginning of the sliding. Charge $X$ to the first event that happens during this sliding, denoted by $Y$. It can be shown that either (i) $Y$ is a 1-level event of the same type as $X$, or (ii) $Y$ is a 1-level or a pseudo-free good event, or (iii) $Y$ is a 1-level or a pseudo-free event of the type illustrated in Fig. 6(c), such that at most one of the $E$-contacts involved in $Y$ is dirty. Repeat this sliding and charging process for each of the three possible choices of $d$. Note that the number of events $Y$ is $O\left(n^{2} \alpha(n)\right)$ in case (ii), and $O\left(n^{2} \alpha(n) \log n\right)$ in case (iii).

Arguing as above, each event $Y$ of type (i) is charged in the fashion described above at most once, and each event of the other types is charged at most a constant number of times. Lemma 3.9 thus implies that the number of events of the Fig. 6(o) type is $O\left(n^{2} \alpha(n) \log n\right)$.

We now handle events of the type illustrated in Fig. 7(f). Fix an $F$-contact $a$ between the (sole) 2 -face of $\mathcal{P}$ and one of the triangles of $\Gamma$. Consider the plane $\Pi_{a}$ defined by this contact as in Section 2.3. Let $\Gamma_{a}$ be the cross section of $\Gamma$ within $\Pi_{a} . \Gamma_{a}$ is a collection of $n$ disjoint segments. Consider their planar Voronoi diagram $\operatorname{Vor}_{\mathcal{P}}\left(\Gamma_{a}\right)$ in the plane $\Pi_{a}$. Its complexity is $O(n)$ [6]. It is easy to see that each free event of $\mathcal{P}$ among $\Gamma$ that involves the contact $a$ uniquely corresponds to a vertex in $\operatorname{Vor}_{\mathcal{P}}\left(\Gamma_{a}\right)$. Since there are $O(n)$ such contacts $a$, we conclude that the overall number of events of the type illustrated in Fig. 7(f) is $O\left(n^{2}\right)$. This completes the proof of the lemma.

Lemma 5.4. $\quad N_{4}=O\left(n^{2+\varepsilon}\right)$, for any $\varepsilon>0$.

Proof. Let $\mathcal{P}$ be a tetrahedron. The events of $\mathcal{P}$ with four active vertices that we analyze (that is, events that are neither good nor segment events) belong to the types illustrated in Figs. 6(b), 6(d), 6(f), 6(h), 6(k), 6(p), 6(r), 7(d), 7(p), 9(i), 10(d), 10(g), and 10(i), or are $V V V V$ events.

Free events of the types shown in Figs. 6(b), 6(k), and 6(p) are LEM events, and their number is thus $O\left(N_{3}\right)=O\left(n^{2} \alpha(n) \log n\right)$ (Lemmas 3.8 and 5.3).

A counting scheme. Let $X$ be a free event of the type illustrated in Fig. 10(g). We show below how to (almost) uniquely charge $X$ either to an $(\leq k)$-level event of a type that was already analyzed, or to $k^{2}(\leq k)$-level events of the type shown in Fig. 10(g), for any fixed parameter $k$. Lemma 3.10 then implies that the number of such events $X$ is $O\left(n^{2+\varepsilon}\right)$, for any $\varepsilon>0$.

The following notation is illustrated in Fig. 11(a). Let $c$ denote the $E$-contact in $X$, and let $d$ denote the $F$-contact in $X$. Let $a$ denote the $V$-contact that is incident to $d$, and let $b$ denote the $V$-contact that is incident to $c$. Let $f_{1}$ denote the (unique) face of $\mathcal{P}$ defined by the three vertices of degree 2 , and let $f_{2}$ be the other face of $\mathcal{P}$ that is incident to the edge involved in $c$. Consider sliding along $a, c$, and $d$, as discussed in Section 2.3. It is easy to see that in one of the two possible directions of this sliding, the only face of $\mathcal{P}$ that belongs to the frontier is $f_{1}$. We slide in this direction. It follows that in any uni-contact event that occurs to $\mathcal{P}$ during the sliding process, the "new" contact 


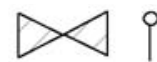

(a) 7, LEM

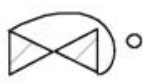

(f) 6, LEM

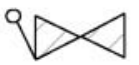

(k) 6, LEM

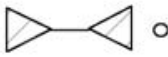

(o) 7, LEM

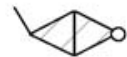

(s) 5 , SFC

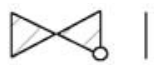

(b) 7, LEM

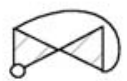

(g) 5 , SFC

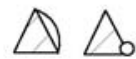

(I) 6 , SFC

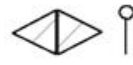

(p) 6, LEM

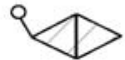

(t) 5 , SFC

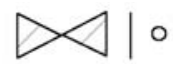

(c) 8, LEM

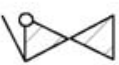

(h) 6, LEM

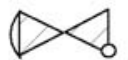

(d) 5 , SFC

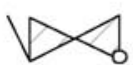

(i) 6, LEM

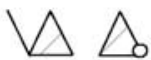

(m) 7, LEM

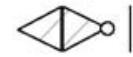

(q) 6 , LEM

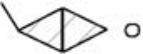

(u) 6, LEM

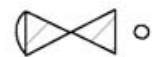

(e) 6, LEM

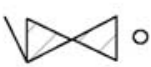

(j) 7, LEM

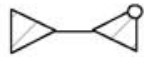

(n) 6, SFC

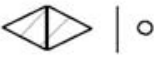

(r) 7, LEM

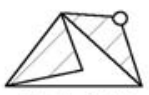

(v) 5 , SFC

Fig. 8. Different combinatorial types of FFEV events, not including good events.<smiles>CC1CC1</smiles>

(a) 6, LEM<smiles>OC1C2C3C1C23</smiles>

(f) 5 , SFC<smiles>OC1CC12CC2</smiles>

(k) 5, LEM

$\Delta \wedge$

(p) 6, LEM

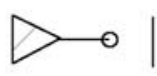

(b) 6, LEM

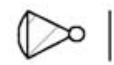

(g) 5, Tag

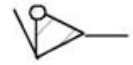

(I) 5 , LEM

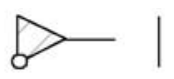

(c) 6, LEM

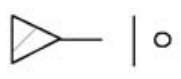

(d) 7, LEM

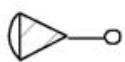

(i) 4 , SEC

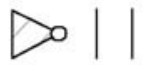

(n) 7, LEM

(m) 6, LEM

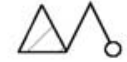

(q) 5 , SFC

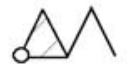

(r) 5, Tag

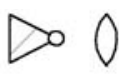

(e) 5 , SFC

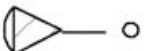

(j) 5, LEM

Fig. 9. Different combinatorial types of FEEV events, not including good events.

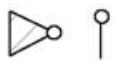

(a) 5 , SFC

Do 。

(f) 5 , LEM

$$
\nabla 0 \mid \circ
$$

(b) 6, LEM

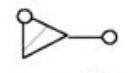

(g) $4, \mathrm{CS}$

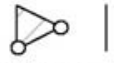

(c) 5 , Tag

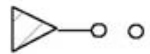

(h) 5, LEM

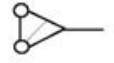

(d) 4 , Sliding

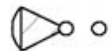

(i) 4 , Tag.

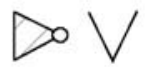

(o) 6, LEM

Fig. 10. Different combinatorial types of FEVV events, not including good events. 


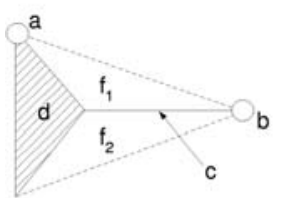

(a)

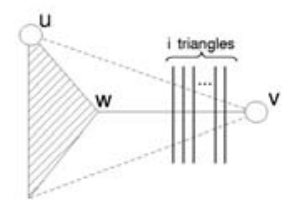

(b)

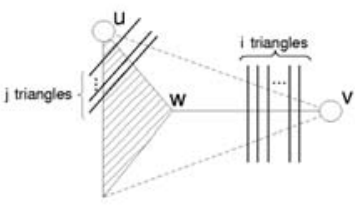

(c)

Fig. 11. An illustration of the counting scheme for the Fig. 10(g) type events. (a) A schematic depiction of the tetrahedron $\mathcal{P}$ with the four contacts involved in such an event. (b) A typical event collected during the first stage, with $i$ triangles of $\Gamma$ separating the vertex $v$ from the other vertices of $\mathcal{P}$. (c) An event collected during the second stage.

(that is not $a, c$, or $d$ ) must involve the face $f_{1}$ or the edges and vertices that lie on its boundary.

Denote the vertices of $f_{1}$ by $u, v$, and $w$, where $u$ and $v$ denote the vertices that are involved in the contacts $a$ and $b$, respectively. Consider the possible types of the "new" contact discussed above. It cannot be a $V$-contact that involves $u$, since $u$ cannot be involved in two different $V$-contacts. It can be a $V$-contact that involves $w$, and in this case the new event is popular. It can be an $E$-contact that involves one of the edges $(u, w)$ or $(v, w)$, or an $F$-contact with the face $f_{1}$, but then $w$ is a popular vertex in these cases as well. If the contact is an $E$-contact that involves the edge $(u, v)$, then the vertex $u$ is popular. If the contact is a $V$-contact that involves $v$, then the event that occurs has the same type as $X$, but might have a higher level. Finally, we can also encounter multi-contact events during the sliding. As follows from Section 2.3, the sliding is well defined before any multi-contact event happens.

Thus, during this sliding we will either encounter events of the same type as $X$ or good events. We slide until we encounter $k$ events of the same type as $X$, or a single good event. If we encounter a good event, we charge $X$ to this event and stop. If we "collect" $k$ events of the same type as $X$, we proceed to a second stage as follows.

Consider any event $X^{\prime}$ from these $k$ collected events and denote the four contacts involved in this event by $a^{\prime}, b^{\prime}, c^{\prime}, d^{\prime}$, in a similar way to the denotation of the contacts involved in $X$. We slide along $b^{\prime}, c^{\prime}$, and $d^{\prime}$, starting at $X^{\prime}$. Consider the sets $\mathcal{P}^{+}$and $\mathcal{P}^{-}$ in this sliding process. We claim that one of these sets contains two-dimensional faces of $\mathcal{P}$, and the other contains one such face. Indeed, by definition the face involved in the $F$-contact $d^{\prime}$ cannot belong to either $\mathcal{P}^{+}$or $\mathcal{P}^{-}$. This leaves three other faces of $\mathcal{P}$, each of which belongs to one of these sets (due to general position); this necessarily implies that one of the sets contains one face and the other set contains two. Assume, without loss of generality, that $\mathcal{P}^{+}$is the set that contains one face. It is easy to see that this face has to be either $f_{1}$ or $f_{2}$, and we assume that it is $f_{1}$. This assumption also incurs no real loss of generality, since the analysis described below is completely analogous when the frontier face is $f_{2}$.

We perform the above sliding along $b^{\prime}, c^{\prime}$, and $d^{\prime}$, in the direction in which $\mathcal{P}^{+}$is the frontier. The only two-dimensional frontier face is $f_{1}$. As in the sliding performed in the first stage, it is easy to see that the events that can be encountered during this sliding are either good events or events of the same type as $X$. As in the first stage, we slide until we either encounter a good event, in which case we charge it, or until we collect $k$ events 
of the type of $X$. It is easy to see that all events that are thus charged or collected have level at most $2 k$. This process is repeated for each of the $k$ events $X^{\prime}$ that are collected in the first stage.

The above described two-stage process either charges $X$ to an $(\leq 2 k)$-level good event or to a collection of $k^{2}(\leq 2 k)$-level events of the same type (it is easily checked that each event charged in the second stage is encountered only once). In order for Lemma 3.10 to imply that the number of events $X$ as above is $O\left(n^{2+\varepsilon}\right)$, for any $\varepsilon>0$, we need to show that each event is charged in this fashion only $O(1)$ times.

Before the first stage of the sliding commences, no triangle intersects the interior of $\mathcal{P}$. With the beginning of the sliding, the triangle that was involved in the contact $b$ either starts to intersect $\mathcal{P}$, or does not. If it does, then it separates the vertex $v$ from the other three vertices of $\mathcal{P}$. The level of the first event that $\mathcal{P}$ encounters in the first stage is thus either 0 or 1 . This first event is either good, in which case we stop the sliding, or it is of the same type as $X$, in which case it involves the contacts $a, c, d$, and a new contact $b^{\prime}$, of the vertex $v$ of $\mathcal{P}$ with some triangle $B$ of $\Gamma$. Notice that before the contact $b^{\prime}$ is reached, the triangle $B$ is disjoint from $\mathcal{P}$, and when $b^{\prime}$ occurs $B$ touches $\mathcal{P}$. Due to linearity of the trajectories, this implies that immediately after the first event occurs, the triangle $B$ intersects $\mathcal{P}$. Moreover, it also separates $v$ from the other three vertices of $\mathcal{P}$. It is easy to see that $B$ cannot stop separating $v$ in this fashion without some (pseudo-free) good event occurring, and $B$ cannot stop intersecting $\mathcal{P}$ by touching the vertex $v$ for the second time, due to the linearity of the trajectories.

We can analogously show that after $i \leq k$ events of the same type as $X$ are collected in the first stage, either $i$ or $i+1$ triangles separate $v$ from the other vertices of $\mathcal{P}$, and no other triangles intersect $\mathcal{P}$ (see Fig. 11(b)). Hence, the level of the $(i+1)$ st collected event is either $i$ or $i+1$. This implies that each event charged in the first stage is charged at most four times: Given any such event $X^{\prime \prime}$, we can reach the event $X$ that charged it by sliding on the contacts $a, c$, and $d$, away from $X^{\prime \prime}$ in one of the two possible directions. The event $X$ is either the first or the second 0-level event that we encounter during this sliding.

The second stage of the sliding is conducted and analyzed similarly, the differences being that the events $X^{\prime}$ from which the sliding processes begin have level $0 \leq i \leq k$ (which is determined by $i$ triangles that separate $v$ from the other vertices of $\mathcal{P}$ ), and that after each event collected during this stage of the sliding, a new triangle of $\Gamma$ begins to separate the vertex $u$ from the other vertices of $\mathcal{P}$ (Fig. 11(c)). For this stage, we can argue that any event is charged at most 16 times.

Indeed, given an event $X^{\prime \prime}$ charged in the second stage, let us slide away from it on the contacts $b, c$, and $d$, in one of the two possible directions. As can be easily verified by the above description of the second stage of the sliding process, the event $X^{\prime}$, from which the second stage of the sliding in which $X^{\prime \prime}$ was charged started, must be either the first or the second event that is encountered during our sliding from $X^{\prime \prime}$, with the property that no triangles separate the vertex $u$ from the other vertices of $\mathcal{P}$ (although some triangles might still separate the vertex $v$ ). We have seen above that each of these four possible events $X^{\prime}$ (two events in each of the two possible directions of sliding from $\left.X^{\prime \prime}\right)$ has at most four events $X$ that can reach it during the first stage of the sliding that commences at $X$. Thus, for any event charged in the second stage, there are at most 16 events $X$ that charge it. 
This concludes the exposition of the counting scheme for free events $X$ of the type illustrated in Fig. 10(g). It is easy to verify that this scheme also works for pseudo-free events of the Fig. $10(\mathrm{~g})$ type, in which only the $F$ - and the $E$-contacts are possibly dirty, and the edge $e$ of the triangle of $\Gamma$ that is involved in the $E$-contact does not intersect the interior of $\mathcal{P}$. This restriction is necessary since in the proof we rely on the fact that the planes $\Pi_{a}, \Pi_{b}, \Pi_{c}$, and $\Pi_{d}$ (see Section 2.3 for definitions) support the tetrahedron $\mathcal{P}$; that is, the interior of $\mathcal{P}$ is completely contained in one of the two half-spaces defined by each plane. This is needed, for instance, when we claim that in one of the directions of the sliding along $a, c$, and $d$, the only frontier face is $f_{1}$-this can only be ensured when $\Pi_{a}, \Pi_{c}$, and $\Pi_{d}$ support $\mathcal{P}$. Now notice that $\Pi_{a}$ and $\Pi_{b}$ support $\mathcal{P}$ only if $a$ and $b$ are clean, $\Pi_{c}$ supports $\mathcal{P}$ only if the above-mentioned edge $e$ does not intersect the interior of $\mathcal{P}$, and $\Pi_{d}$ always supports $\mathcal{P}$, even when the $F$-contact $d$ is dirty.

Sliding and charging. Let $X$ be a free event of the type illustrated in Fig. 9(i). Let $a$ denote the edge of $\mathcal{P}$ that is involved in an $E$-contact, and lies on the face that is involved in the $F$-contact. Use the SEC technique (Section 3.7) to slide away from the $E$-contact that involves $a$. Charge $X$ to the first event $Y$ encountered during the sliding. $Y$ is a free event, since the placements of $\mathcal{P}$ immediately upon the beginning of the sliding are free. The SEC technique implies that $Y$ cannot have an $E$-contact that involves $a$. This implies that either (i) $Y$ is a good free event, or (ii) $Y$ is a free event of the type shown in Fig. $10(\mathrm{~g})$. Since the charging of $X$ to $Y$ is unique, the number of events of the type illustrated in Fig. 9(i) is $O\left(n^{2+\varepsilon}\right)$, for any $\varepsilon>0$.

Note that this analysis also applies to pseudo-free events of the Fig. 9(i) type in which only the $E$-contacts are possibly dirty, and the edge $e$ of a triangle of $\Gamma$ that is involved in such an $E$-contact does not intersect the interior of $\mathcal{P}$. Notice that when we start the above sliding from a pseudo-free event $X$ and charge an event $Y$ of the type shown in Fig. 10(g), $Y$ may also be pseudo-free. The above restriction on the dirtyness of the contacts in $X$ ensures that if $Y$ is a pseudo-free event, it fits the requirements set above for pseudo-free Fig. $10(\mathrm{~g})$ type events, which ensures that the number of such events $Y$ can be bounded by $O\left(n^{2+\varepsilon}\right)$, for any $\varepsilon>0$.

Let $X$ be a free event of the type illustrated in Fig. 10(d). Denote the $F$-, the $E$-, and one of the $V$-contacts involved in $X$ by $a, b$, and $c$, respectively. Slide on $a, b$, and $c$, as described in Section 2.3, such that the placement of $\mathcal{P}$ is free immediately after the beginning of the sliding, and charge $X$ to the first event $Y$ that occurs during the sliding. $Y$ is clearly a free event. Moreover, it is easy to see (arguing as in the analysis of type Fig. $10(\mathrm{~g}))$ that the frontier of the sliding consists of the face of $\mathcal{P}$ that is incident to the $V$-contact $c$ and the $E$-contact $b$, plus the edges and vertices incident to this face. Since a fourth contact in $Y$ can only involve frontier features (Section 2.3), we conclude that $Y$ is either a good event or an event of the Fig. 10(g) type. Since the charging of $X$ to $Y$ is unique, the number of events $X$ as above is $O\left(n^{2+\varepsilon}\right)$, for any $\varepsilon>0$.

Note that this analysis also applies to pseudo-free events of the Fig. 10(d) type in which only the $F$ - and the $E$-contacts are possibly dirty, and the edge $e$ of a triangle of $\Gamma$ that is involved in the $E$-contact does not intersect the interior of $\mathcal{P}$. As in the case of type Fig. 9(i) events, this restriction is necessary to ensure that the number of charged pseudo-free events of type Fig. $10(\mathrm{~g})$ can be bounded by $O\left(n^{2+\varepsilon}\right)$, for any $\varepsilon>0$. 
The Tagansky technique. We treat events of the type illustrated in Fig. 7(p) using the Tagansky technique. Consider a free event $X$ that belongs to this type. Let $d$ be one of the two $V$-contacts involved in $X$. Denote the other three contacts by $a, b$, and $c$. Slide on the contacts $a, b$, and $c$, such that the triangle of $\Gamma$ that is involved in $d$ starts intersecting $\mathcal{P}$ immediately upon the beginning of the sliding. Charge $X$ to the first event that happens during this sliding, denoted by $Y$. It can be shown that either (i) $Y$ is a 1-level event of the same type as $X$, or (ii) $Y$ is a pseudo-free or a 1-level good event. Repeat this sliding and charging process for each of the two possible choices of $d$. Note that the number of events $Y$ in case (ii) is $O\left(n^{2} \alpha(n)\right)$. Each event $Y$ of type (i) is charged in the fashion described above at most once, and each event of the other types is charged at most a constant number of times. Lemma 3.9 thus implies that the number of events of the Fig. 7(p) type is $O\left(n^{2} \alpha(n) \log n\right)$.

Let $X$ be a free event of the type illustrated in Fig. 10(i). Let $d$ be one of the two $V$-contacts involved in $X$. Denote the other three contacts by $a, b$, and $c$. Slide on the contacts $a, b$, and $c$, such that the triangle of $\Gamma$ that is involved in $d$ starts intersecting $\mathcal{P}$ immediately upon the beginning of the sliding. Charge $X$ to the first event that happens during this sliding, denoted by $Y$. It can be shown that either (i) $Y$ is a 1-level event of the same type as $X$, or (ii) $Y$ is a pseudo-free or a 1-level good event, or (iii) $Y$ is an event of the Fig. 9(i) type, such that either $Y$ is a 1-level free event or $Y$ is a pseudo-free event in which only the $E$-contacts are possibly dirty, and the edge $e$ of a triangle of $\Gamma$ that is involved in such a dirty contact does not intersect the interior of $\mathcal{P}$. Repeat this sliding and charging process for each of the two possible choices of $d$. Each event $Y$ of type (i) is charged in the fashion described above at most once, and each event of the other types is charged at most a constant number of times. Lemma 3.9 thus implies that the number of events $X$ as above is $O\left(n^{2+\varepsilon}\right)$, for any $\varepsilon>0$. Note that this analysis also applies to pseudo-free events of the Fig. 10(i) type in which only the $E$-contact is dirty, and the edge $e$ of the triangle of $\Gamma$ that is involved in this contact does not intersect the interior of $\mathcal{P}$.

Let $X$ be a free event of the type illustrated in Fig. 6(d) (see also Fig. 12(a)). Let $d$ be one of the two $E$-contacts involved in $X$, and let $f$ be the corresponding edge of $\mathcal{P}$. Denote the other three contacts by $a, b$, and $c$. Slide on the contacts $a, b, c$ as above, and charge $X$ to the first event that happens during this sliding, denoted by $Y$, as long as this event is not a pseudo-free event of the same type as $X$. That is, when we encounter a pseudo-free event of the same type as $X$ we simply keep sliding "as if nothing happened". Such pseudo-free events arise when the triangle $\delta$ of $\Gamma$, involved in the contact $d$ and now intersecting $\mathcal{P}$, touches $f$ with another edge (as shown in Fig. 12(b)). We ignore such events simply because we do not have a bound on their number, and the Tagansky technique cannot be used to charge free events to 0-level pseudo-free events of the same type. Charging such events would thus not help us. Ignoring them, on the other hand, does not cause any difficulty - only one such event can happen before an event that can be charged is reached during the sliding, and, as argued below, the charging is unique.

It can be shown that either (i) $Y$ is a 1-level event of the same type as $X$, or (ii) $Y$ is a pseudo-free or a 1-level good event, or (iii) $Y$ is a pseudo-free or a 1-level LEM event, or (iv) $Y$ is a pseudo-free or a 1-level event of the type illustrated in Fig. 10(d), such that only the $F$-contact is possibly dirty, or (v) $Y$ is a 1-level free event of the type illustrated in Fig. 10(i). Repeat this sliding and charging process for each of the 


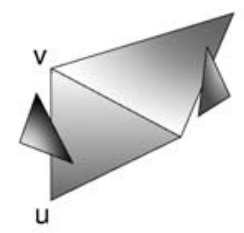

(a)

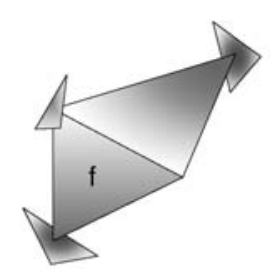

(d)

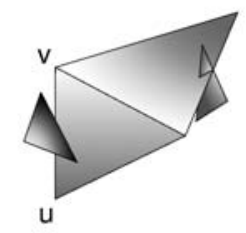

(b)

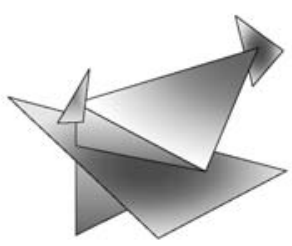

(e)

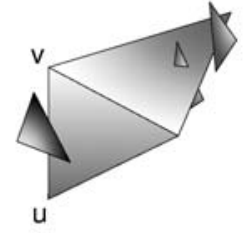

(c)

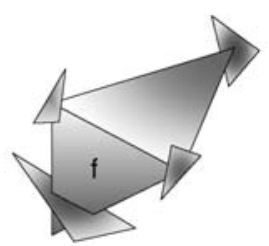

(f)

Fig. 12. The analysis of Fig. 6(d) events is illustrated in (a)-(c). (a) A three-dimensional rendition of such an event, in which the tetrahedron $\mathcal{P}$ is shown touching two triangles of $\Gamma$ in two $E$-contacts. The $V$-contacts involving the vertices $u$ and $v$ are not shown. (b) A pseudo-free event that can be reached during the analysis. (c) A charged 1-level event. A similar illustration of the analysis of Fig. 7(d) events is given in (d)-(f). (d) One such event, depicting the three $V$-contacts, but not showing the $F$-contact involving the face $f$. (e) A pseudo-free event that can be reached during the analysis. (f) A charged 1-level event.

two possible choices of $d$. Each event $Y$ of type (i) is charged in the fashion described above at most once (see below), and each event of the other types is charged at most a constant number of times. Lemma 3.9 thus implies that the number of events $X$ as above is $O\left(n^{2+\varepsilon}\right)$, for any $\varepsilon>0$.

The uniqueness of charging of events $Y$ of type (i) is argued in analogy to the analysis of type Fig. 2(b), which was treated in Lemma 4.3. Similarly to that case, the triangle $\delta$ keeps intersecting both of the faces of $\mathcal{P}$ incident to the edge $f$ until charging is performed. Given a charged 1-level event $Y$ (illustrated in Fig. 12(c)), the pair of faces intersected by the penetrating triangle allows us to determine uniquely the free event $X$ that charged $Y$. Indeed, we can reach $X$ by "letting go" of the $E$-contact involving the edge shared by these two faces (a unique such contact must exist), and sliding in the direction in which the triangle of $\Gamma$ involved in this contact does not start to penetrate $\mathcal{P}$. Notice that the fact that a pseudo-free event of the same type as $X$ may happen during the sliding is irrelevant to this uniqueness argument. This completes the analysis of Fig. 6(d) events.

Let $X$ be a free event of the type illustrated in Fig. 7(d) (see also Fig. 12(d)). Let $d$ be one of the two $V$-contacts that are incident to the $F$-contact involved in $X$, and let $v$ be the corresponding vertex of $\mathcal{P}$. Denote the other three contacts by $a, b$, and $c$. Slide on the contacts $a, b, c$ as above, and charge $X$ to the first event that happens during this sliding, denoted by $Y$, as long as this event is not a pseudo-free event of the same type as $X$. Here, again (as for Fig. 6(d) events), if such a pseudo-free event is encountered, we ignore it and keep sliding. Such a pseudo-free event happens if the triangle $\delta$ of $\Gamma$, involved in $d$ and now intersecting $\mathcal{P}$, sweeps a new vertex of $\mathcal{P}$ (see Fig. 12(e)). As in 
the case of Fig. 6(d) events, it is easy to see that only at most one such pseudo-free event can happen during the sliding and ignoring such events does not cause any difficulty.

It can be shown that either (i) $Y$ is a 1-level event of the same type as $X$, or (ii) $Y$ is a pseudo-free or a 1-level good event, or (iii) $Y$ is a 1-level free event of the Fig. 7(p) type, or (iv) $Y$ is a pseudo-free or a 1-level event of one of the types illustrated in Figs. 10(g) and 10(i), such that only the $E$-contact is possibly dirty, and the edge $e$ of the triangle of $\Gamma$ that is involved in this contact does not intersect the interior of $\mathcal{P}$. Repeat this sliding and charging process for each of the two possible choices of $d$. Each event $Y$ of type (i) is charged in the fashion described above at most twice.

We cannot argue that each 1-level event $Y$ of type (i) is charged at most once because there are events $Y$ of this type for which there is no unique way to determine the charging event $X$. One such event $Y$ is illustrated in Fig. 12(f). In the situation depicted in this figure there are two $V$-contacts incident to the face $f$, and to reach $X$ we have to let go of one of them and slide on the other three contacts. However, there is no way to determine which $V$-contact should be dropped, so there are two "equally good" ways of sliding away from $Y$, and consequently there may be two free events $X$ that charge $Y$.

If, for both possible choices of the contact $d$ above, we have reached an event of type (i), then we have only managed to charge two 1-level events that may be both charged twice. This is not sufficient for the Tagansky technique to work, so we proceed to perform a second type of charging. Let $d$ be the $V$-contact involved in $X$ that is not incident to any other contact. Denote the other three contacts by $a, b$, and $c$, and perform sliding and charging as usual (there is no need to treat pseudo-free events of the same type as $X$ in a special way, since it is easily verified that such an event cannot be the first event encountered during the sliding). It can be shown that the event $Y$ that is charged by $X$ is now either (i) a 1-level event of the same type as $X$, or (ii) a pseudo-free or a 1-level good event, or (iii) a 1-level or a pseudo-free event of the type illustrated in Fig. 10(d), such that only the $E$-contact is possibly dirty, and the edge $e$ of the triangle of $\Gamma$ that is involved in this contact does not intersect the interior of $\mathcal{P}$, or (iv) a pseudo-free or a 1-level event of the type illustrated in Fig. 7(f). Moreover, it is easy to check that each event $Y$ of type (i) is now charged at most once, and each event of the other types is charged at most a constant number of times.

We have described two stages of sliding and charging for free events $X$ of the Fig. 7(d) type. In the first stage we have charged $X$ either to an event from a class of events whose size is $O\left(n^{2+\varepsilon}\right)$, for any $\varepsilon>0$, or to two 1-level events of the same type as $X$, such that each 1-level event is charged in this fashion at most twice (in the terminology of Section 3.10, $i_{1}=2$ and $j_{1}=2$ ). In the second stage we were able to charge $X$ either to an event from a class of events of size $O\left(n^{2+\varepsilon}\right)$, for any $\varepsilon>0$, or to one 1-level event of the same type as $X$, such that each 1-level event is uniquely charged in this fashion $\left(i_{2}=1\right.$ and $\left.j_{2}=1\right)$. Thus, $i_{1} / j_{1}+i_{2} / j_{2}=2$, and Lemma 3.9 implies that the number of events $X$ as above is $O\left(n^{2+\varepsilon}\right)$, for any $\varepsilon>0$.

Let $X$ be a free event of one of the types illustrated in Figs. 6(f) and 6(r). Let $d$ be either the $V$-contact involved in $X$, or the $E$-contact that is incident to a vertex of degree 1 in $X$. Denote the other three contacts by $a, b$, and $c$. Slide on the contacts $a, b, c$ as above, and charge $X$ to the first event that happens during this sliding, denoted by $Y$, as long as this event is not a pseudo-free event of the same type as $X$. It can be shown that 
either (i) $Y$ is a 1-level event of the same type as $X$, or (ii) $Y$ is a pseudo-free or a 1-level good event, or (iii) $Y$ is a pseudo-free or a 1-level LEM event, or (iv) $Y$ is a pseudo-free or a 1-level event with three active vertices, or (v) $Y$ is a pseudo-free or a 1-level EEEE event. Repeat this sliding and charging process for each of the two possible choices of $d$. Each event $Y$ of type (i) is charged in the fashion described above at most once, and each event of the other types is charged at most a constant number of times. Lemma 3.9 thus implies that the number of events $X$ as above is $O\left(n^{2} \alpha(n) \log ^{2} n\right)$.

Let $X$ be a free event of the Fig. 6(h) type. Let $d$ be one of the $E$-contacts involved in $X$. Denote the other three contacts by $a, b$, and $c$. Slide on the contacts $a, b, c$ as above, and charge $X$ to the first event that happens during this sliding, denoted by $Y$, as long as this event is not a pseudo-free event of the same type as $X$. It can be shown that either (i) $Y$ is a 1-level event of the same type as $X$, or (ii) $Y$ is a pseudo-free or a 1-level good event, or (iii) $Y$ is a pseudo-free or a 1-level LEM event, or (iv) $Y$ is a pseudo-free or a 1-level event with three active vertices, or (v) $Y$ is a pseudo-free or a 1-level event of the type illustrated in Fig. $10(\mathrm{~g})$, such that only the $F$-contact is possibly dirty. Repeat this sliding and charging process for each of the two possible choices of $d$. Each event $Y$ of type (i) is charged in this fashion at most once (uniqueness of charging is argued in complete analogy to the case of type Fig. 6(d)), and each event of the other types is charged at most a constant number of times. Lemma 3.9 thus implies that the number of events $X$ as above is $O\left(n^{2+\varepsilon}\right)$, for any $\varepsilon>0$.

$V V V V$ events. Finally, let $X$ be a free $V V V V$ event. Choose three of the contacts involved in $X$. Denote these contacts by $a, b$, and $c$, and refer to the fourth contact as $d$. Slide on the contacts $a, b$, and $c$, such that the triangle of $\Gamma$ that is involved in $d$ starts intersecting $\mathcal{P}$ immediately upon the beginning of the sliding. Charge $X$ to the first event that happens during this sliding, denoted by $Y$. It can be shown that either (i) $Y$ is a 1-level $V V V V$ event, or (ii) $Y$ is a pseudo-free or a 1-level LEM event, or (iii) $Y$ has three active vertices, or (iv) $Y$ is a 1-level free event of the type illustrated in Fig. 7(d), or (v) $Y$ is a 1-level good event. Repeat this sliding and charging process for each of the four possible triples $(a, b, c)$. The charging of $X$ to $Y$ is easily seen to be unique. The Tagansky technique thus implies that the number of $V V V V$ events is $O\left(n^{2+\varepsilon}\right)$, for any $\varepsilon>0$. This, finally, completes the proof of the lemma.

Lemma 5.5. $N_{5}=O\left(n^{2+\varepsilon}\right)$, for any $\varepsilon>0$.

Proof. Let $\mathcal{P}$ be a convex polyhedron with five vertices. The events of $\mathcal{P}$ with five active vertices that we analyze belong to the types illustrated in Figs. 6(a), 6(e), 6(i), 6(m), 6(q), 6(s), 6(t), 7(e), 7(i), 7(l), 7(q), 8(d), 8(g), 8(s), 8(t), 8(v), 9(e), 9(f), 9(g), 9(j), 9(k), 9(1), 9(q), 9(r), 9(s), 10(a), 10(c), 10(f), 10(h), and 10(j).

Free events of the types shown in Figs. 6(a), 6(e), 6(i), 6(m), 6(s), 6(t), 7(e), 7(q), 9(j), 9(k), 9(1), 9(s), 10(f), 10(h), and 10(j) are LEM events, and their number is thus $O\left(N_{4}\right)=O\left(n^{2+\varepsilon}\right)$, for any $\varepsilon>0$ (Lemmas 3.8 and 5.4).

During the rest of the proof, we rely on the specific geometric structure of the pentahedron $\mathcal{P}$, as visualized in Figs. 13(a) and 13(b). Recall from Section 2.1 that we assume $\mathcal{P}$ to be simplicial. This implies that $\mathcal{P}$ must be the union of two tetrahedra with a common base. It has two "extreme" vertices of degree 3 that are not connected by an 


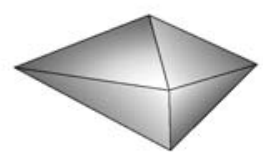

(a)

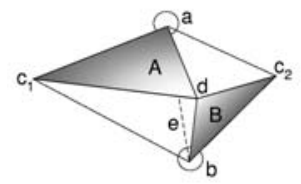

(d)

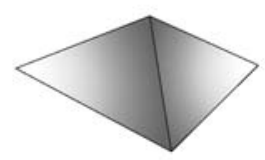

(b)

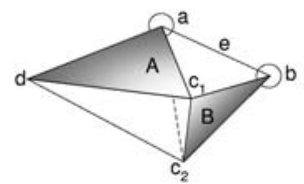

(e)

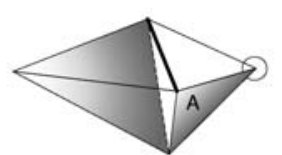

(c)

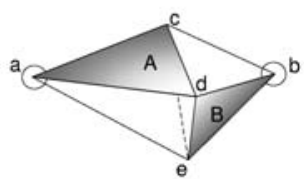

(f)

Fig. 13. The geometry of the pentahedron is visualized in (a) and (b), which show views from two opposing directions. (c) The only possible geometric realization (up to symmetry) of an event of the type shown in Fig. $8(\mathrm{~g})$; the wire-frame of the pentahedron is depicted, with the two faces that participate in the $F$-contacts shown filled, and the edge that participates in the $E$-contact thickened; the vertex involved in the $V$-contact is marked by a circle. (d)-(f) The three possible geometric realizations of an event of the Fig. 7(i) type. Again, the two faces that participate in the $F$-contacts are filled, and the vertices involved in the $V$-contacts are marked by circles.

edge, between which there is a "belt" of three vertices of degree 4. $\mathcal{P}$ has six triangular faces, each incident to one "extreme" vertex and two "belt" vertices.

After this brief geometric description we can proceed with the analysis. Let $X$ be a free event of a type illustrated in Fig. 9(e), 9(f), 9(q), or 10(a). Use the SFC technique to slide away from the $F$-contact of $X$, as in the proof of Lemma 4.4. Charge $X$ to the first event $Y$ encountered during the sliding. Either (i) $Y$ is good, or (ii) $Y$ is a LEM event, or (iii) $Y$ has at most four active vertices. Since the charging of $X$ to $Y$ is unique, the number of events of the types illustrated in Figs. 10(a), 9(e), 9(f), and 9(q) is $O\left(n^{2+\varepsilon}\right)$, for any $\varepsilon>0$.

Events of the Fig. 8(t) and the Fig. 8(v) types are handled in an analogous fashion, by sliding away from the $F$-contact that is incident to a vertex of degree 1 .

Events of the Fig. 8(g) type are similarly handled by sliding away from the $F$-contact that is incident to the $V$-contact. In these cases the event $Y$ that is charged may also belong to one of the types illustrated in Figs. 9(q) and 9(s), but the number of these events is still $O\left(n^{2+\varepsilon}\right)$, for any $\varepsilon>0$.

It might not be clear why, say, an event of the Fig. 8(s) type cannot be reached in the above sliding. Indeed, if we think of the events purely combinatorially, as they are illustrated in Fig. 8, this seems possible. However, it cannot happen due to the geometric structure of $\mathcal{P}$. It is easy to see that, without loss of generality, a Fig. 8(g) type event has to be realized as shown in Fig. 13(c). (In particular, the $E$-contact has to involve a "belt" edge of $\mathcal{P}$, since otherwise the two $F$-contacts and the $E$-contact would have to be incident to a common face, which would have made it popular.) Consider the above sliding process in light of this realization; we let go of the $F$-contact $A$ (see Fig. 13(c)) and try to "replace" it with a new contact. Looking at the figure we can see that if this 
new contact is an $F$-contact, some vertex or face becomes popular. If the new contact is a $V$-contact, then the event has to be a LEM event or good. Finally, if the fourth contact is an $E$-contact, the only "interesting" types of events that may occur are the ones illustrated in Figs. 9(q) and 9(s).

Keeping the geometric constraints imposed by the structure of $\mathcal{P}$ in mind, we proceed to treat events of the types illustrated in Figs. 8(d) and 8(s). They are handled in analogy to the Fig. $8(\mathrm{~g})$ type (that is, by sliding away from the $F$-contact that is incident to the $V$-contact). Conducting a similar geometric analysis, we can see that the event $Y$ that is charged may belong to one of the types illustrated in Figs. 9(f) and 9(q). Since the number of these events is known to be $O\left(n^{2+\varepsilon}\right)$, for any $\varepsilon>0$, we conclude that this bound also holds for the events we are treating.

The bounds derived above also hold for pseudo-free events of the discussed types.

The Tagansky technique. Let $X$ be a free event of the type illustrated in Fig. 9(r). Let $d$ be either the $V$-contact involved in $X$ or the $E$-contact that is incident to a vertex of $\mathcal{P}$ that has degree 1 . Denote the other three contacts by $a, b$, and $c$. Slide on the contacts $a$, $b$, and $c$ such that the triangle of $\Gamma$ that is involved in $d$ starts intersecting $\mathcal{P}$ immediately upon the beginning of the sliding. Charge $X$ to the first event that happens during this sliding, denoted by $Y$, as long as this event is not a pseudo-free event of the same type as $X$. It can be shown that either (i) $Y$ is a 1-level event of the same type as $X$, or (ii) $Y$ is a pseudo-free or a 1-level good event, or (iii) $Y$ is a pseudo-free or a 1-level LEM event, or (iv) $Y$ is a pseudo-free or a 1-level event with four active vertices, or (v) $Y$ is a pseudo-free or a 1-level segment event, or (vi) $Y$ is a pseudo-free or a 1-level event of the type illustrated in Fig. 8(v) or 9(q). Repeat this sliding and charging process for each of the two possible choices of $d$. Each event $Y$ of type (i) is charged in this fashion at most once, and each event of the other types is charged at most a constant number of times. Lemma 3.9 thus implies that the number of events $X$ as above is $O\left(n^{2+\varepsilon}\right)$, for any $\varepsilon>0$. Note that this analysis also applies to pseudo-free events of the Fig. 9(r) type in which only the $F$-contact and the $E$-contact that is incident to the $F$-contact are possibly dirty.

Events $X$ of the Fig. 9(g) type are handled analogously. The difference is that $d$ is chosen to be either the $V$-contact or the $E$-contact that is not incident to any other contact involved in $X$, and that the event $Y$ is either (i) a 1-level event of the same type as $X$, or (ii) a pseudo-free or a 1-level good event, or (iii) a pseudo-free or a 1-level segment event, or (iv) a pseudo-free or a 1-level event with four active vertices, or (v) a pseudo-free or a 1-level event of the type illustrated in Fig. 9(f).

Sliding and charging. Let $X$ be a free event of the type illustrated in Fig. 7(i). We distinguish between two possibilities. The first is that the two vertices of $\mathcal{P}$ that are involved in the two $V$-contacts are connected by an edge of $\mathcal{P}$. This means that either they are both belt vertices of $\mathcal{P}$ (see Fig. 13(d)) or one is a belt vertex and the other is an extreme vertex (Fig. 13(e)). The other possibility is that these two vertices are not adjacent along an edge, which implies that they are both extreme vertices of $\mathcal{P}$ (Fig. 13(f)).

Assume first that the vertices are adjacent. In this case we use the SFC technique (Section 3.6) to slide away from one of the two $F$-contacts, and charge the first event we encounter. The SFC technique implies that this event does not involve an $F$-contact 
with the same face. We prove below that this event either has four active vertices, or it is a good or a LEM event, or it is of the type that is illustrated in Fig. 10(a). This implies that the number of events $X$ that fall under the above assumption is $O\left(n^{2+\varepsilon}\right)$, for any $\varepsilon>0$.

The following notation is illustrated in Figs. 13(d) and 13(e). Denote the two vertices involved in the $V$-contacts by $a$ and $b$, and the two faces involved in the $F$-contacts by $A$ and $B$, with $a \in A$ and $b \in B$. Denote the edge that connects $a$ to $b$ by $e$. $e$ is adjacent to two triangular faces, $f_{1}$ and $f_{2}$. $f_{1}$ has vertices $a, b$, and a third vertex denoted by $c_{1}$. Denote the symmetric vertex of $f_{2}$ by $c_{2}$.

As mentioned above, either (i) both $a$ and $b$ are belt vertices, in which case $a, b, c_{1}$, $c_{2}$ are all connected by respective edges to the fifth vertex, denoted by $d$ (see Fig. 13(d)), or (ii) only one of $a, b$, say $a$, is a belt vertex, in which case $c_{1}$ and $c_{2}$ are connected by an edge, and the fifth vertex $d$ is connected to $c_{1}, c_{2}$, and $a$ (Fig. 13(e)).

Assume that case (i) holds. $c_{1}$ and $c_{2}$ are not connected by an edge, and both of them are incident to three edges and three faces of $\mathcal{P}$. Since $A$ is incident to $a$ but not to $b$, it must be either $a c_{1} d$ or $a c_{2} d$, and, similarly, $B$ is either $b c_{1} d$ or $b c_{2} d$. Hence $d$ is the common vertex of $A$ and $B$, and, without loss of generality, $A=a c_{1} d$ and $B=b c_{2} d$. Therefore, both $c_{1}$ and $c_{2}$ have degree 1 , and lie on exactly one face that is involved in an $F$-contact.

Consider sliding away from the contact that involves $A$, and charging a new event $Y$ as described above. Assume that this event is not good. If the vertex $c_{1}$ is not active in this event, the event has four active vertices. If $c_{1}$ is involved in a $V$-contact, then $Y$ is a LEM event. If $c_{1}$ is incident to an $E$-contact, then $Y$ is either a LEM event, or an event of the type that is illustrated in Fig. 10(a). If $c_{1}$ is incident to an $F$-contact with some face $C$, then either $C=c_{1} a b$ or $C=c_{1} b d$ ( $C$ cannot be $c_{1} d a=A$ since we have slid away from $A$ ). In both cases $b$ is a popular vertex, contrary to assumption. Hence, $Y$ is either good, or a LEM event, or an event of the type that is illustrated in Fig. 10(a). This proves our claim, and concludes the treatment of events of the Fig. 7(i) type in which the vertices that are involved in the $V$-contacts are connected by an edge and case (i) holds. The analysis for case (ii) is very similar. In this case we must have $B=b c_{1} c_{2}$ and $A$ is either $a c_{1} d$ or $a c_{2} d$, say $A=a c_{1} d$ (Fig. 13(e)). Here we slide away from $B$, and verify easily that the charged event $Y$ must be of one of the asserted types.

Consider now an event $X$ of the Fig. 7(i) type in which the vertices $a, b$ that are involved in the $V$-contacts (denoted by $V_{1}, V_{2}$, respectively) are the extreme vertices of $\mathcal{P}$ and are thus not connected by an edge (see Fig. 13(f)). Denote the three belt vertices by $c, d, e$, and let the faces involved in the $F$-contacts be $A=a c d, B=b d e$, without loss of generality (Fig. 13(f)). Denote the two respective $F$-contacts by $\alpha$ and $\beta$, and consider the line $l=\Pi_{\alpha} \cap \Pi_{\beta}$, using the notation from Section 2.3. The vertex $d$ lies on this line and partitions it into two rays, $r_{1}$ and $r_{2}$. The polytope $\mathcal{P}$ has six faces, and the two faces that are involved in the $F$-contacts are not visible from any point on the line $l$ (using again definitions from Section 2.3). The other four faces are dae, aec, ecb, and $c b d$.

Slide along $\alpha, \beta$, and $V_{1}$. In one of the two possible directions of the sliding, dae is the only frontier face. (That is, either $\mathcal{P}^{+}$or $\mathcal{P}^{-}$contain dae and no other 2-face of $\mathcal{P}$.) Indeed, assume for the sake of contradiction that $\mathcal{P}^{+}$contains dae and at least one other face. (The case of $\mathcal{P}^{-}$containing dae is symmetric.) Since $\mathcal{P}^{+}$cannot have singular 
vertices, at least one other face that shares an edge with dae has to belong to $\mathcal{P}^{+}$. The face $a d c$, that shares the edge $a d$ with $d a e$, is involved in an $F$-contact and cannot belong to $\mathcal{P}^{+}$, and similarly for the face $e d b$. Thus, the face $a e c$ belongs to $\mathcal{P}^{+}$. Consider now the frontier $\mathcal{P}^{+}$as seen from the point $p=p_{\alpha, \beta, V_{1}}$. This point lies on the line $l$, and sees the frontier as a convex polygon in which the vertex $a$ has to be strictly convex, because $a$ is involved in the $V$-contact $V_{1}$. However, since $p$ lies on $\Pi_{\alpha}$ and $\mathcal{P}^{+}$contains the edges $d a$ and $a c$, which also both lie on $\Pi_{\alpha}$, the points $d, a$, and $c$ are collinear and $a$ lies between $d$ and $c$, when seen from $p$. Hence, $a$ is not a strictly convex vertex of $\mathcal{P}^{+}$, which brings us to a contradiction, as desired.

We can thus perform the above sliding such that the only frontier face is dae, and charge $X$ to the first encountered event $Y . Y$ either has a multi-contact or a new unicontact that involves dae or one of its edges or faces. It is easy to see that $Y$ is either good or a Fig. 7(i) type event in which the $V$-contacts are adjacent along an edge of $\mathcal{P}$. The number of events of the latter kind was proved above to be $O\left(n^{2+\varepsilon}\right)$, for any $\varepsilon>0$. This concludes the analysis of Fig. 7(i) type events, and shows that their number is $O\left(n^{2+\varepsilon}\right)$, for any $\varepsilon>0$.

Let $X$ be a free event of the type illustrated in Fig. 7(1). As above, consider the two $F$-contacts $\alpha$ and $\beta$, and one of the $V$-contacts, denoted by $V_{1}$. Let the faces involved in $\alpha$ and $\beta$ be $a c d$ and bed, respectively, and let the vertex involved in $V_{1}$ be $a$. For the right choice of $V_{1}$, we can slide along $\alpha, \beta$, and $V_{1}$, as above, such that the only frontier face is dae. We charge to the first event $Y$ encountered during the sliding, and, as above, this event is either good or is of the Fig. 7(i) type. This shows that the number of Fig. 7(1) type events is $O\left(n^{2+\varepsilon}\right)$, for any $\varepsilon>0$.

Note that the above analysis of Fig. 7(i) and Fig. 7(1) type events also holds for pseudo-free such events in which only the $F$-contacts are possibly dirty.

The Tagansky technique again. Let $X$ be a free event of the type illustrated in Fig. 10(c). Let $d$ be one of the two $V$-contacts involved in $X$. Denote the other three contacts by $a$, $b$, and $c$. Slide on the contacts $a, b, c$, such that the triangle of $\Gamma$ that is involved in $d$ starts intersecting $\mathcal{P}$ immediately upon the beginning of the sliding. Charge $X$ to the first event that happens during this sliding, denoted by $Y$, as long as this event is not a pseudo-free event of the same type as $X$. It can be shown that either (i) $Y$ is a 1-level event of the same type as $X$, or (ii) $Y$ is a pseudo-free or a 1-level good event, or (iii) $Y$ is a 1-level free event of the types illustrated in Figs. 8(d), 8(s), and 9(e), or (iv) $Y$ is a pseudo-free or a 1-level event of the type illustrated in Fig. 10(a), or (v) $Y$ is a pseudo-free or a 1-level event of one of the types illustrated in Figs. 9(r) and 9(g), such that only the $F$-contact and the $E$-contact that is incident to the $F$-contact are possibly dirty. Repeat this sliding and charging process for each of the two possible choices of $d$. Each event $Y$ of type (i) is charged in the fashion described above at most twice, arguing as for the type of Fig. 7(d).

Now let $d$ be the $E$-contact involved in $X$. Denote the other three contacts by $a, b$, and $c$, and perform sliding as above. Charge the first event $Y$ encountered during the sliding, as long as this event is not a pseudo-free event of the same type as $X$. It can be shown that the event $Y$ that is charged by $X$ is now either (i) a 1-level event of the same type as $X$, or (ii) a pseudo-free or a 1-level good event, or (iii) a pseudo-free or a 1-level event with at most four active vertices, or (iv) a 1-level free or a pseudo-free event of the 
type illustrated in Fig. 7(1), such that only the $F$-contacts are possibly dirty. Each event $Y$ of type (i) is charged in this fashion at most once, and each event of the other types is charged at most a constant number of times.

We have described two stages of sliding and charging for free events $X$ of the Fig. 10(c) type. In the terminology of Section $3.10, i_{1}=j_{1}=2$ and $i_{2}=j_{2}=1$. Thus, $i_{1} / j_{1}+$ $i_{2} / j_{2}=2$, and Lemma 3.9 implies that the number of events $X$ as above is $O\left(n^{2+\varepsilon}\right)$, for any $\varepsilon>0$.

Events of the type illustrated in Fig. 6(q) are also treated using the Tagansky technique. Consider a free event $X$ that belongs to this type. Choose three of the contacts involved in $X$. Denote these contacts by $a, b$, and $c$, and refer to the fourth contact as $d$. Slide on the contacts $a, b$, and $c$, such that the triangle of $\Gamma$ that is involved in $d$ starts intersecting $\mathcal{P}$ immediately upon the beginning of the sliding. Charge $X$ to the first event that happens during this sliding, denoted by $Y$, as long as this event is not a pseudo-free event of the same type as $X$. It can be shown that either (i) $Y$ is a 1-level event of the same type as $X$, or (ii) $Y$ is a pseudo-free or a 1-level LEM event, or (iii) $Y$ has at most four active vertices, or (iv) $Y$ is a 1-level event of the type illustrated in Fig. 10(c), or (v) $Y$ is a 1-level good event. Repeat this sliding and charging process for each of the four possible triples $(a, b, c)$. The charging of $X$ to $Y$ is easily seen to be unique. The Tagansky technique thus implies that the number of events of the type illustrated in Fig. 6(q) is determined by the number of events of the Fig. 10(c) type, and is thus $O\left(n^{2+\varepsilon}\right)$, for any $\varepsilon>0$.

Lemma 5.6. $N_{6}=O\left(n^{2+\varepsilon}\right)$, for any $\varepsilon>0$.

Proof. Let $\mathcal{P}$ be a convex polyhedron with six vertices. The events of $\mathcal{P}$ with six active vertices that we analyze belong to the types illustrated in Figs. 6(g), 6(j), 6(1), 7(a), 7(b), 7(j), 7(o), 7(r), 7(u), 8(e), 8(f), 8(h), 8(i), 8(k), 8(l), 8(n), 8(p), 8(q), 8(u), 9(a), 9(b), 9(c), 9(h), 9(m), 9(o), 9(p), 10(b), and 10(e).

Free events of the types shown in Figs. 6(g), 6(l), 7(j), 7(r), 7(u), 8(e), 8(f), 8(h), 8(i), 8(k), 8(p), 8(q), 8(u), 9(a), 9(b), 9(c), 9(h), 9(m), 9(o), 9(p), 10(b), and 10(e) are LEM events, and their number is thus $O\left(N_{5}\right)=O\left(n^{2+\varepsilon}\right)$, for any $\varepsilon>0$ (Lemmas 3.8 and 5.5).

We treat events of the type illustrated in Fig. 6(j) using the Tagansky technique in the same way as for events of the Fig. 6(q) type. It can be shown that the events $Y$ that are charged are either (i) 1-level events of the same type as $X$, or (ii) pseudo-free or 1-level LEM events, or (iii) events with at most five active vertices, or (iv) 1-level good events. The Tagansky technique implies that the number of events of the type illustrated in Fig. 6(j) is $O\left(N_{5}\right)=O\left(n^{2+\varepsilon}\right)$, for any $\varepsilon>0$.

Let $X$ be a free event of a type illustrated in Fig. 7(a) or 7(b). Use the SFC technique to slide away from the $F$-contact that is incident only to one other $F$-contact and to no $V$-contacts. Charge $X$ to the first event $Y$ encountered during the sliding. Either (i) $Y$ is good, or (ii) $Y$ is a LEM event, or (iii) $Y$ has at most five active vertices. Since the charging of $X$ to $Y$ is unique, the number of events of the types illustrated in Figs. 7(a) and 7(b) is $O\left(n^{2+\varepsilon}\right)$, for any $\varepsilon>0$.

Events of the Fig. 8(n) type are handled in an analogous fashion, by sliding away from the $F$-contact that is incident only to the $E$-contact, and not incident to the $V$-contact. Events of the types illustrated in Figs. 7(o) and 8(1) are handled by sliding away from any of the $F$-contacts. The number of events of these types is also $O\left(n^{2+\varepsilon}\right)$, for any $\varepsilon>0$. 
Lemma 5.7. $N_{7}=O\left(n^{2+\varepsilon}\right)$, for any $\varepsilon>0$.

Proof. Let $\mathcal{P}$ be a convex polyhedron with seven vertices. The events of $\mathcal{P}$ with seven active vertices that we analyze belong to the types illustrated in Figs. 6(n), 7(c), 7(g), 7(k), 7(n), 7(s), 7(t), 8(a), 8(b), 8(j), 8(m), 8(o), 8(r), 9(d), and 9(n). All of these events except for the Fig. 6(n) type are LEM events, and the number of free such events is $O\left(N_{6}\right)=O\left(n^{2+\varepsilon}\right)$, for any $\varepsilon>0$ (Lemmas 3.8 and 5.6).

We treat events of the type illustrated in Fig. 6(n) using the Tagansky technique in the same way as for events of the Fig. 6(q) type. It can be shown that the events $Y$ that are charged are either (i) 1-level events of the same type as $X$, or (ii) pseudo-free or 1-level LEM events, or (iii) events with at most six active vertices, or (iv) 1-level good events. The Tagansky technique thus implies that the number of events of the type illustrated in Fig. 6(n) is $O\left(N_{6}\right)=O\left(n^{2+\varepsilon}\right)$, for any $\varepsilon>0$.

Lemma 5.8. $\quad N_{8}=O\left(n^{2+\varepsilon}\right)$, for any $\varepsilon>0$.

Proof. Let $\mathcal{P}$ be a convex polyhedron with eight vertices. The events of $\mathcal{P}$ with eight active vertices that we analyze belong to the types illustrated in Figs. 7(h), 7(m), and 8(c). All of these events are LEM events, and the number of free such events is thus $O\left(N_{7}\right)=$ $O\left(n^{2+\varepsilon}\right)$, for any $\varepsilon>0$ (Lemmas 3.8 and 5.7).

Lemma 5.9. $N_{d}=O\left(n^{2+\varepsilon}\right)$, for any $\varepsilon>0$, for $9 \leq d \leq 12$.

Proof. Any event with $9 \leq d \leq 12$ active vertices is either a good or a segment event. As described above, the number of such events is easily bounded by $O\left(n^{2+\varepsilon}\right)$, for any $\varepsilon>0$.

This completes the proof of Theorem 5.1.

\section{Approximate Nearest-Neighbor Searching}

Theorem 5.1 can be applied to obtain the following result.

Theorem 6.1. Let $\delta>0$ be a fixed parameter. We can preprocess a collection of disjoint polyhedra in 3-space with $n$ vertices altogether into a data structure of size $O\left(n^{2+\varepsilon} / \delta^{4}\right)$, for any $\varepsilon>0$, such that this data structure can answer $\delta$-approximate Euclidean nearest-neighbor queries amidst the polyhedra in time $O(\log (n / \delta))$.

The data structure described in the theorem is essentially a point-location data structure on a polyhedral Voronoi diagram of the collection of polyhedra ("sites"). We use the fact that the Euclidean ball $\mathcal{B}$ in $\mathbb{R}^{3}$ can be $\delta$-approximated by a convex polytope $\mathcal{P}$ with $O(1 / \delta)$ vertices; that is, $\mathcal{P} \subseteq \mathcal{B} \subseteq(1+\delta) \mathcal{P}$ [13]. It is easy to verify that the Euclidean distance of any point $x \in \mathbb{R}^{3}$ from its closest site under the distance function 
induced by $\mathcal{P}$ is at most $1+\delta$ times the Euclidean distance of $x$ to its closest site under the Euclidean metric. Therefore, $\delta$-approximate Euclidean nearest-neighbor queries amidst the sites can be answered using point location queries in the polyhedral Voronoi diagram of the sites under the distance function induced by $\mathcal{P}$. Theorem 5.1 states that the complexity of this diagram is $O\left(n^{2+\varepsilon} / \delta^{4}\right)$, for any $\varepsilon>0$, and standard machinery [28, Section 8.3.3] can be used to preprocess it into a point location data structure with the desired performance bounds.

The algorithm described in [28] goes back to [8]. It constructs a point-location data structure for a three-dimensional subdivision, using top-down sampling of a decomposition of the subdivision into cells of constant description complexity [28]. Roughly speaking, if the subdivision can be decomposed into $N$ such cells, the size of the constructed data structure is $O\left(N^{1+\varepsilon}\right)$, for any $\varepsilon>0$.

Any Voronoi diagram of complexity $R$ in three dimensions can be decomposed into $O(R)$ cells of constant description complexity, due to the star-shapedness of each Voronoi cell with respect to its defining site [22]. To decompose a Voronoi cell, one can project each feature of the cell (of dimension $d-2$ or lower) onto the site, and construct decomposition surfaces along all the projection rays. Repeating this construction for every cell decomposes the whole Voronoi diagram into sub-cells which can then be decomposed further into cells of constant description complexity. We omit the easy details. Since Theorem 5.1 implies that the described polyhedral Voronoi diagram has complexity $O\left(n^{2+\varepsilon} / \delta^{4}\right)$, for any $\varepsilon>0$, it can be decomposed into $O\left(n^{2+\varepsilon} / \delta^{4}\right)$ cells. Consequently, a point location structure of size $O\left(n^{2+\varepsilon} / \delta^{4}\right)$, for any $\varepsilon>0$, can be constructed using the method of [8] and [28].

Remark. We have also developed an alternative solution to this problem, designing a data structure of size only $O\left(n^{2+\varepsilon} / \delta\right)$. However, the query time of this data structure is $O((\log n) / \delta)$. Roughly speaking, it performs $O(1 / \delta)$ logarithmic-time queries of several types, among which are ray-shooting queries from the query point in the fixed directions of the vertices of $\mathcal{P}$ (as seen from the center of $\mathcal{P}$ ).

\section{Acknowledgements}

Part of the work on this paper has been carried out while the first author was visiting the University of Illinois at Urbana-Champaign, hosted by Sariel Har-Peled. The author is grateful to Sariel for the hospitality and for many helpful discussions. In particular, the results reported in Section 6 were developed in collaboration with Sariel.

\section{References}

1. P. K. Agarwal, O. Schwarzkopf, and M. Sharir. The overlay of lower envelopes and its applications. Discrete Comput. Geom., 15:1-13, 1996.

2. P. K. Agarwal and M. Sharir. Arrangements and their applications. In J.-R. Sack and J. Urrutia, editors, Handbook of Computational Geometry, pages 49-119. Elsevier Science/North-Holland, Amsterdam, 2000 . 
3. P. K. Agarwal and M. Sharir. Pipes, cigars, and kreplach: the union of Minkowski sums in three dimensions. Discrete Comput. Geom., 24:645-685, 2000.

4. B. Aronov and M. Sharir. On translational motion planning of a convex polyhedron in 3-space. SIAM J. Comput., 26:1785-1803, 1997.

5. B. Aronov, M. Sharir, and B. Tagansky. The union of convex polyhedra in three dimensions. SIAM J. Comput., 26:1670-1688, 1997.

6. F. Aurenhammer and R. Klein. Voronoi diagrams. In J.-R. Sack and J. Urrutia, editors, Handbook of Computational Geometry, pages 201-290. Elsevier Science/North-Holland, Amsterdam, 2000.

7. J.-D. Boissonnat, M. Sharir, B. Tagansky, and M. Yvinec. Voronoi diagrams in higher dimensions under certain polyhedral distance functions. Discrete Comput. Geom., 19(4):473-484, 1998.

8. B. Chazelle and M. Sharir. An algorithm for generalized point location and its applications. J. Symbolic Comput., 10:281-309, 1990.

9. L. P. Chew. Near-quadratic bounds for the $L_{1}$ Voronoi diagram of moving points. Comput. Geom. Theory Appl., 7:73-80, 1997.

10. L. P. Chew, K. Kedem, M. Sharir, B. Tagansky, and E. Welzl. Voronoi diagrams of lines in 3-space under polyhedral convex distance functions. J. Algorithms, 29(2):238-255, 1998.

11. K. L. Clarkson and P. W. Shor. Applications of random sampling in computational geometry, II. Discrete Comput. Geom., 4:387-421, 1989.

12. T. K. Dey and W. Zhao. Approximate medial axis as a Voronoi subcomplex. In Proc. 7th ACM Symposium on Solid Modeling and Applications, pages 356-366, 2002.

13. R. M. Dudley. Metric entropy of some classes of sets with differentiable boundaries. J. Approx. Theory, 10(3):227-236, 1974.

14. H. Edelsbrunner and R. Seidel. Voronoi diagrams and arrangements. Discrete Comput. Geom., 1:25-44, 1986.

15. S. Fortune. Voronoi diagrams and Delaunay triangulations. In J. E. Goodman and J. O'Rourke, editors, Handbook of Discrete and Computational Geometry, chapter 20, pages 377-388. CRC Press, Boca Raton, FL, 1997.

16. D. Halperin and M. Sharir. New bounds for lower envelopes in three dimensions, with applications to visibility in terrains. Discrete Comput. Geom., 12:313-326, 1994.

17. S. Har-Peled. A replacement for Voronoi diagrams of near linear size. In Proc. 42nd IEEE Symposium on Foundations of Computer Science, pages 94-103, 2001.

18. C. Icking and L. Ma. A tight bound for the complexity of Voronoi diagrams under polyhedral convex distance functions in 3D. In Proc. 33rd ACM Symposium on the Theory of Computing, pages 316-321, 2001.

19. V. Koltun. Almost tight upper bounds for vertical decompositions in four dimensions. In Proc. 42nd IEEE Symposium on Foundations of Computer Science, pages 56-65, 2001.

20. V. Koltun and M. Sharir. On the overlay of envelopes in four dimensions. In Proc. 13th ACM-SIAM Symposium on Discrete Algorithms, pages 810-819, 2002.

21. V. Koltun and M. Sharir. Three dimensional Euclidean Voronoi diagrams of lines with a fixed number of orientations. In Proc. 18th ACM Symposium on Computational Geometry, pages 217-226, 2002.

22. D. Leven and M. Sharir. Intersection and proximity problems and Voronoi diagrams. In J. T. Schwartz and C.-K. Yap, editors, Advances in Robotics 1: Algorithmic and Geometric Aspects of Robotics, pages 187-228. Erlbaum, Hillsdale, NJ, 1987.

23. A. Okabe, B. Boots, K. Sugihara, and S. N. Chiu. Spatial Tessellations: Concepts and Applications of Voronoi Diagrams, 2nd edition. Wiley, New York, 2000.

24. O. Schwarzkopf and M. Sharir. Vertical decomposition of a single cell in a three-dimensional arrangement of surfaces and its applications. Discrete Comput. Geom., 18:269-288, 1997.

25. M. I. Shamos. The early years of computational geometry - a personal memoir. In B. Chazelle, J. E. Goodman, and R. Pollack, editors, Advances in Discrete and Computational Geometry, pages 313-333. American Mathematical Society, Providence, RI, 1998.

26. M. I. Shamos and D. Hoey. Closest-point problems. In Proc. 16th IEEE Symposium on Foundations of Computer Science, pages 151-162, 1975.

27. M. Sharir. Almost tight upper bounds for lower envelopes in higher dimensions. Discrete Comput. Geom., 12:327-345, 1994. 
28. M. Sharir and P. K. Agarwal. Davenport-Schinzel Sequences and Their Geometric Applications. Cambridge University Press, New York, 1995.

29. B. Tagansky. The Complexity of Substructures in Arrangements of Surfaces. Ph.D. thesis, Tel Aviv University, Tel Aviv, 1996.

30. B. Tagansky. A new technique for analyzing substructures in arrangements of piecewise linear surfaces. Discrete Comput. Geom., 16:455-479, 1996.

Received July 11, 2002, and in revised form April 21, 2003. Online publication November 5, 2003. 